\title{
Near-Exact Distributions for Likelihood Ratio Statistics Used in the Simultaneous Test of Conditions on Mean Vectors and Patterns of Covariance Matrices
}

\author{
Carlos A. Coelho, ${ }^{1,2}$ Filipe J. Marques, ${ }^{1,2}$ and Sandra Oliveira ${ }^{1,3}$ \\ ${ }^{1}$ Centro de Matemática e Aplicações (CMA), FCT/UNL, 2829-516 Caparica, Portugal \\ ${ }^{2}$ Departamento de Matemática, Faculdade de Ciências e Tecnologia, Universidade Nova de Lisboa, 2829-516 Caparica, Portugal \\ ${ }^{3}$ Departamento de Economia e Gestão, Instituto Politécnico de Setúbal, 2910-761 Setúbal, Portugal
}

Correspondence should be addressed to Filipe J. Marques; fjm@fct.unl.pt

Received 31 May 2015; Accepted 23 November 2015

Academic Editor: Andrzej Swierniak

Copyright ( 2016 Carlos A. Coelho et al. This is an open access article distributed under the Creative Commons Attribution License, which permits unrestricted use, distribution, and reproduction in any medium, provided the original work is properly cited.

\begin{abstract}
The authors address likelihood ratio statistics used to test simultaneously conditions on mean vectors and patterns on covariance matrices. Tests for conditions on mean vectors, assuming or not a given structure for the covariance matrix, are quite common, since they may be easily implemented. But, on the other hand, the practical use of simultaneous tests for conditions on the mean vectors and a given pattern for the covariance matrix is usually hindered by the nonmanageability of the expressions for their exact distribution functions. The authors show the importance of being able to adequately factorize the c.f. of the logarithm of likelihood ratio statistics in order to obtain sharp and highly manageable near-exact distributions, or even the exact distribution in a highly manageable form. The tests considered are the simultaneous tests of equality or nullity of means and circularity, compound symmetry, or sphericity of the covariance matrix. Numerical studies show the high accuracy of the near-exact distributions and their adequacy for cases with very small samples and/or large number of variables. The exact and near-exact quantiles computed show how the common chi-square asymptotic approximation is highly inadequate for situations with small samples or large number of variables.
\end{abstract}

\section{Introduction}

Testing conditions on mean vectors is a common procedure in multivariate statistics. Often a given structure is assumed for the covariance matrix, without testing it, or otherwise this test to the covariance structure is carried out apart. This is often due to the fact that the exact distribution of the test statistics used to test simultaneously conditions on mean vectors and patterns on covariance matrices is too elaborate to be used in practice. The authors show how this problem may be overcome with the development of very sharp and manageable near-exact distributions for the test statistics. These distributions may be obtained from adequate factorizations of the characteristic function (c.f.) of the logarithm of the likelihood ratio (l.r.) statistics used for these tests.

The conditions tested on mean vectors are

(i) the equality of all the means in the mean vector, (ii) the nullity of all the means in the mean vector and the patterns tested on covariance matrices are

(i) circularity,

(ii) compound symmetry,

(iii) sphericity.

Let $\underline{X}=\left[X_{1}, \ldots, X_{p}\right]^{\prime}$ be a random vector with $\operatorname{Var}(\underline{X})=$ $\Sigma_{c}$. The covariance matrix $\Sigma_{c}$ is said to be circular, or circulant, if $\Sigma_{c}=\left[\sigma_{i j}\right], i, j=1, \ldots, p$, with

$$
\begin{aligned}
\sigma_{i i} & =\operatorname{Var}\left(X_{i}\right)=\sigma_{0}^{2}, \\
\sigma_{i, i+k} & =\sigma_{i+k, i}=\operatorname{Cov}\left(X_{i}, X_{i+k}\right)=\sigma_{0}^{2} \rho_{k},
\end{aligned}
$$

where $\rho_{k}=\rho_{p-k}=\operatorname{Corr}\left(X_{i}, X_{i+k}\right)$, for $i=1, \ldots, p ; k=1, \ldots$, $p-i$. 
For example, for $p=6$ and $p=7$, we have

$$
\begin{gathered}
\Sigma_{c}=\sigma_{0}^{2}\left[\begin{array}{llllll}
1 & \rho_{1} & \rho_{2} & \rho_{3} & \rho_{2} & \rho_{1} \\
\rho_{1} & 1 & \rho_{1} & \rho_{2} & \rho_{3} & \rho_{2} \\
\rho_{2} & \rho_{1} & 1 & \rho_{1} & \rho_{2} & \rho_{3} \\
\rho_{3} & \rho_{2} & \rho_{1} & 1 & \rho_{1} & \rho_{2} \\
\rho_{2} & \rho_{3} & \rho_{2} & \rho_{1} & 1 & \rho_{1} \\
\rho_{1} & \rho_{2} & \rho_{3} & \rho_{2} & \rho_{1} & 1
\end{array}\right], \\
\Sigma_{c}=\sigma_{0}^{2}\left[\begin{array}{lllllll}
1 & \rho_{1} & \rho_{2} & \rho_{3} & \rho_{3} & \rho_{2} & \rho_{1} \\
\rho_{1} & 1 & \rho_{1} & \rho_{2} & \rho_{3} & \rho_{3} & \rho_{2} \\
\rho_{2} & \rho_{1} & 1 & \rho_{1} & \rho_{2} & \rho_{3} & \rho_{3} \\
\rho_{3} & \rho_{2} & \rho_{1} & 1 & \rho_{1} & \rho_{2} & \rho_{3} \\
\rho_{3} & \rho_{3} & \rho_{2} & \rho_{1} & 1 & \rho_{1} & \rho_{2} \\
\rho_{2} & \rho_{3} & \rho_{3} & \rho_{2} & \rho_{1} & 1 & \rho_{1} \\
\rho_{1} & \rho_{2} & \rho_{3} & \rho_{3} & \rho_{2} & \rho_{1} & 1
\end{array}\right] .
\end{gathered}
$$

Besides the almost obvious area of times series analysis, there is a wealth of other areas and research fields where circular or circulant matrices arise, such as statistical signal processing, information theory and cryptography, biological sciences, psychometry, quality control, and signal detection, as well as spatial statistics and engineering, when observations are made on the vertices of a regular polygon.

We say that a positive-definite $p \times p$ covariance matrix $\Sigma_{c s}$ is compound-symmetric if we can write

$$
\begin{aligned}
& \Sigma_{c s}=b E_{p p}+(a-b) I_{p}=a I_{p}+b\left(E_{p p}-I_{p}\right) \\
& \text { with }-\frac{a}{(p-1)}<b<a .
\end{aligned}
$$

For example, for $p=4$, we have

$$
\Sigma_{c s}=\left[\begin{array}{llll}
a & b & b & b \\
b & a & b & b \\
b & b & a & b \\
b & b & b & a
\end{array}\right] .
$$

If, in (3), $b=0$, we say that the matrix is spheric.

The 1.r. tests for equality and nullity of means, assuming circularity, and the l.r. tests for the simultaneous test of equality or nullity of means and circularity of the covariance matrix were developed by [1], while the test for equality of means, assuming compound symmetry, and the test for equality of means and compound symmetry were formulated by [2] and the test for nullity of the means, assuming compound symmetry, and the simultaneous test for nullity of the means and compound symmetry of the covariance matrix were worked out by [3]. The exact distribution for the l.r. test statistic for the simultaneous test of equality of means and circularity of the covariance matrix was obtained in [4] and is briefly referred to in Section 2, for the sake of completeness, while near-exact distributions for the l.r. test statistic for the simultaneous test of nullity of the means and circularity of the covariance matrix are developed in Section 3. Near-exact distributions for the l.r. test statistics for the simultaneous test of equality and nullity of the means and compound symmetry of the covariance matrix are developed in Sections 4 and 5, using a different approach from the one used in Section 3. The 1.r. statistics for the tests of equality and nullity of all means, assuming sphericity of the covariance matrix, may be analyzed in Appendix C and the l.r. statistics for the simultaneous tests of equality and nullity of all means and sphericity, together with the development of near-exact distributions for these statistics, may be examined in Sections 6 and 7 .

Since, as referred above, the exact distributions for the statistics for the simultaneous tests of conditions on means vectors and patterns of covariance matrices are too elaborate to be used in practice, the authors propose in this paper the use of near-exact distributions for these statistics. These are asymptotic distributions which are built using a different concept in approximating distributions which combines an adequately developed decomposition of the c.f. of the statistic or of its logarithm, most often a factorization, with the action of keeping then most of this c.f. unchanged and replacing the remaining smaller part by an adequate asymptotic approximation $[5,6]$. All this is done in order to obtain a manageable and very well-fitting approximation, which may be used to compute near-exact quantiles or $p$ values. These distributions are much useful in situations where it is not possible to obtain the exact distribution in a manageable form and the common asymptotic distributions do not display the necessary precision. Near-exact distributions show very good performances for very small samples, and when correctly developed for statistics used in Multivariate Analysis, near-exact distributions display a sharp asymptotic behavior both for increasing sample sizes and for increasing number of variables.

In Sections 3-7, near-exact distributions are obtained using different techniques and results, according to the structure of the exact distribution of the statistic.

In order to study, in each case, the proximity between the near-exact distributions developed and the exact distribution, we will use the measure

$$
\Delta=\frac{1}{2 \pi} \int_{-\infty}^{+\infty}\left|\frac{\Phi_{W}(t)-\Phi_{W}^{*}(t)}{t}\right| d t
$$

with

$$
\max _{w}\left|F_{W}(w)-F_{W}^{*}(w)\right|=\max _{\ell}\left|F_{\Lambda}(\ell)-F_{\Lambda}^{*}(\ell)\right| \leq \Delta,
$$

where $\Lambda$ represents the l.r. statistic, $\Phi_{W}(t)$ is the exact c.f. of $W=-\log \Lambda, \Phi_{W}^{*}(t)$ is the near-exact c.f., and $F_{W}(\cdot), F_{\Lambda}(\cdot)$, $F_{W}^{*}(\cdot)$, and $F_{\Lambda}^{*}(\cdot)$ are the exact and near-exact c.d.f.s of $W$ and $\Lambda$.

This measure is particularly useful, since in our cases we do not have the exact c.d.f. of $\Lambda$ or $W$ in a manageable form, but we have both the exact and near-exact c.f.'s for $W=$ $-\log \Lambda$. 


\section{The Likelihood Ratio Test for the Simultaneous Test of Equality of Means and the Circularity of the Covariance Matrix}

Let $\underline{X} \sim N_{p}(\mu, \Sigma)$, where $\mu=\left[\mu_{1}, \ldots, \mu_{p}\right]^{\prime}$. Then, for a sample of size $n$, the $(2 / n)$ th power of the l.r. statistic to test the null hypothesis

$$
\begin{gathered}
H_{0}: \mu_{1}=\cdots=\mu_{p} ; \\
\Sigma=\Sigma_{c},
\end{gathered}
$$

is

$$
\begin{aligned}
\Lambda_{1} & =2^{2(p-m-1)} n^{p} \frac{|A|}{v_{1}} \prod_{j=2}^{p} \frac{1}{v_{j}+w_{j}} \\
& =2^{2(p-m-1)} \frac{|V|}{v_{1}} \prod_{j=2}^{p} \frac{1}{v_{j}+w_{j}},
\end{aligned}
$$

where $m=\lfloor p / 2\rfloor, A$ is the maximum likelihood estimator (m.l.e.) of $\Sigma, V=n U^{\prime} A U$, where $U$ is the matrix with running element

$$
\begin{aligned}
& u_{i j}=\frac{1}{\sqrt{p}}\left\{\cos \left(\frac{2 \pi(j-1)(i-1)}{p}\right)+\sin \left(\frac{2 \pi(j-1)(i-1)}{p}\right)\right\}, \\
& v_{j}= \begin{cases}v_{j j}, & j=1, \text { and also } j=m+1 \text { if } p \text { is even, } \\
v_{j j}+v_{p-j+2, p-j+2}, & j=2, \ldots, m, \text { and also } j=m+1 \text { if } p \text { is odd, }\end{cases}
\end{aligned}
$$

with $v_{j}=v_{p-j+2}(j=2, \ldots, m)$, and where $v_{j j}$ is the $j$ th diagonal element of $V$, and

$$
\begin{aligned}
& w_{j} \\
& = \begin{cases}y_{j}^{2}, & j=1, \text { and also } j=m+1 \text { if } p \text { is even, } \\
y_{j}^{2}+y_{p-j+2}^{2}, & j=2, \ldots, m, \text { and also } j=m+1 \text { if } p \text { is odd, }\end{cases}
\end{aligned}
$$

with $\underline{Y}=\left[y_{j}\right]=\sqrt{n} \underline{\bar{X}} U$, where $\underline{\bar{X}}$ is the vector of sample means.

This test statistic was derived by [1, sec. 5.2], where the expression for the l.r. test statistic has to be slightly corrected.

According to [1],

$$
\Lambda_{1} \stackrel{d}{\equiv} \prod_{j=2}^{p} Y_{j}
$$

where

$$
Y_{j} \sim \begin{cases}\operatorname{Beta}\left(\frac{n-j}{2}, \frac{j}{2}\right), & j=2, \ldots, m+1, \\ \operatorname{Beta}\left(\frac{n-j}{2}, \frac{j+1}{2}\right), & j=m+2, \ldots, p,\end{cases}
$$

are a set of $p-1$ independent r.v.s.

From this fact we may write the c.f. of $W_{1}=-\log \Lambda_{1}$ as

$$
\begin{gathered}
\Phi_{W_{1}}(t)=E\left(e^{i t W_{1}}\right)=E\left(e^{-i t \log \Lambda_{1}}\right)=E\left(\Lambda_{1}^{-i t}\right) \\
=\prod_{j=2}^{m+1} \frac{\Gamma(n / 2) \Gamma((n-j) / 2-i t)}{\Gamma((n-j) / 2) \Gamma(n / 2-i t)} \\
\cdot \prod_{j=m+2}^{p} \frac{\Gamma((n+1) / 2) \Gamma((n-j) / 2-i t)}{\Gamma((n-j) / 2) \Gamma((n+1) / 2-i t)} .
\end{gathered}
$$

By adequately handling this c.f., the exact distribution of $W_{1}$ is obtained in [4] as a Generalized Integer Gamma (GIG) distribution (see [7] for the GIG distribution), since we may write

$$
\Phi_{W_{1}}(t)=\prod_{j=1}^{p}\left(\frac{n-j}{2}\right)^{r_{j}}\left(\frac{n-j}{2}-i t\right)^{-r_{j}}
$$

for

$$
\begin{aligned}
& r_{j} \\
& = \begin{cases}\frac{p-2+p \bmod 2}{2}, & j=1, \\
\frac{p-p \bmod 2}{2}-\left\lfloor\frac{j-1-p \bmod 2}{2}\right\rfloor, & j=2, \ldots, p .\end{cases}
\end{aligned}
$$

A popular asymptotic approximation for the distribution of $n W_{1}$ is the chi-square asymptotic distribution with a number of degrees of freedom equal to the difference of the number of unknown parameters under the alternative hypothesis and the number of parameters under the null hypothesis, which gives for $n W_{1}=-n \log \Lambda_{1}$, for $\Lambda_{1}$ in (8), a chi-square asymptotic distribution with $p(p+3) / 2-$ $\lfloor(p+2) / 2\rfloor-1$ degrees of freedom. Although this is a valid approximation for large sample sizes, in practical terms, this approximation is somewhat useless given the fact that it gives quantiles that are much lower than the exact ones, as it may be seen from the quantiles in Table 1, namely, for small samples or when the number of variables involved is somewhat large.

From the values in Table 1 we may see that even for quite large sample sizes and rather small number of variables as in the case of $p=10$ and $n=460$, the asymptotic chisquare quantile does not even match the units digit of the exact quantile, a difference that gets even larger as the number of variables increases. The chi-square asymptotic quantiles are always smaller than the exact ones, their use leading to an excessive number of rejections of the null hypotheses, a problem that becomes a grievous one when we use smaller samples or larger numbers of variables. 
TABLE 1: Exact and asymptotic 0.95 and 0.99 quantiles for $n W_{1}$ where $W_{1}=-\log \Lambda_{1}$ for the statistic $\Lambda_{1}$ in (8), for different values of $p$ and samples of size $n=p+1,50,450$.

\begin{tabular}{|c|c|c|c|}
\hline$p$ & $n$ & exact & Asymptotic- $\chi^{2}$ \\
\hline \multicolumn{4}{|c|}{$\alpha=0.95$} \\
\hline \multirow[t]{4}{*}{10} & & & 76.77780315606147980433710659 \\
\hline & 11 & 184.84579506364826855487849906 & \\
\hline & 60 & 82.86779631112725385496956047 & \\
\hline & 460 & 77.50088072977322094345813820 & \\
\hline \multirow[t]{4}{*}{15} & & & 153.19790274395621072198817490 \\
\hline & 16 & 356.83946609433702153375390686 & \\
\hline & 65 & 169.23132191434840041430238602 & \\
\hline & 465 & 155.17420633635277455721974156 & \\
\hline \multirow[t]{4}{*}{25} & & & 379.74587752919253597245376194 \\
\hline & 26 & 853.62442647392551959929457598 & \\
\hline & 75 & 437.12290346767321994020024210 & \\
\hline & 475 & 387.31925318201483716457949700 & \\
\hline \multirow[t]{4}{*}{50} & & & 1382.92839770564012472044120417 \\
\hline & 51 & 2983.52950629554250120199516974 & \\
\hline & 100 & 1719.07640203276757900109720368 & \\
\hline & 500 & 1434.09183007253302711800352147 & \\
\hline & & $\alpha=0.99$ & \\
\hline \multirow[t]{4}{*}{10} & & & 85.95017624510346845181671517 \\
\hline & 11 & 221.13637373719535956938670312 & \\
\hline & 60 & 92.78317859393323169599466291 & \\
\hline & 460 & 86.75984117402977424037787646 & \\
\hline \multirow[t]{4}{*}{15} & & & 165.84100085082047675645088502 \\
\hline & 16 & 409.92566639020778120425384446 & \\
\hline & 65 & 183.23718212829228159346647123 & \\
\hline & 465 & 167.98095654076846741881112565 & \\
\hline \multirow[t]{4}{*}{25} & & & 399.22970790268112734530953113 \\
\hline & 26 & 940.55141434060365229501805667 & \\
\hline & 75 & 459.68274728064743270409254333 & \\
\hline & 475 & 407.19370031104569525049581690 & \\
\hline \multirow[t]{4}{*}{50} & & & 1419.46244733465596475819616876 \\
\hline & 51 & 3156.01716925813527651187643029 & \\
\hline & 100 & 1765.17588807596249988258749774 & \\
\hline & 500 & 1471.99072215013613268320536543 & \\
\hline
\end{tabular}

\section{The Likelihood Ratio Test for the Simultaneous Test of Nullity of Means and the Circularity of the Covariance Matrix}

For a sample of size $n$, the $(2 / n)$ th power of the l.r. test statistic to test the null hypothesis

$$
\begin{aligned}
H_{0}: \underline{\mu} & =\underline{0} ; \\
\Sigma & =\Sigma_{c}
\end{aligned}
$$

is

$$
\begin{aligned}
\Lambda_{2} & =2^{2(p-m-1)} n^{p} \frac{|A|}{v_{1}} \prod_{j=1}^{p} \frac{1}{v_{j}+w_{j}} \\
& =2^{2(p-m-1)} \frac{|V|}{v_{1}} \prod_{j=1}^{p} \frac{1}{v_{j}+w_{j}},
\end{aligned}
$$

where $m, v_{j}$, and $w_{j}$, as well as the matrices $A$ and $V$, are defined as in the previous section.

According to [1],

$$
\Lambda_{2} \stackrel{d}{\equiv} \prod_{j=1}^{p} Y_{j}
$$

where

$$
Y_{j} \sim \begin{cases}\operatorname{Beta}\left(\frac{n-j}{2}, \frac{j}{2}\right), & j=1, \ldots, m+1, \\ \operatorname{Beta}\left(\frac{n-j}{2}, \frac{j+1}{2}\right), & j=m+2, \ldots, p\end{cases}
$$

are a set of $p$ independent r.v.s. 
Taking $W_{2}=-\log \Lambda_{2}$ and following similar steps to the ones used in [4] to handle the c.f. of $W_{1}$, we may write the c.f. of $W_{2}$ as

$$
\begin{aligned}
\Phi_{W_{2}}(t)= & \frac{\Gamma(n / 2) \Gamma((n-1) / 2-i t)}{\Gamma((n-1) / 2) \Gamma(n / 2-i t)} \\
& \cdot \prod_{j=1}^{p}\left(\frac{n-j}{2}\right)^{r_{j}}\left(\frac{n-j}{2}-i t\right)^{-r_{j}},
\end{aligned}
$$

for $r_{j}$ given by (15).

This shows that the exact distribution of $W_{2}$ is the same as that of the sum of GIG distributions of depth $p$ with an independent Logbeta $((n-1) / 2,1 / 2)$ distributed r.v.

But then, using the result in expression (3) of [8], we know that we can replace asymptotically a $\operatorname{Logbeta}(a, b)$ distribution by an infinite mixture of $\Gamma(b-2 j, a+(b-1) / 2)$ distributions $(j=0,1, \ldots)$, for large values of $a$. This means that we can replace asymptotically

$$
\begin{aligned}
& \frac{\Gamma(n / 2) \Gamma((n-1) / 2-i t)}{\Gamma((n-1) / 2) \Gamma(n / 2-i t)} \\
& \text { by } \sum_{j=0}^{\infty} \pi_{j}\left(\frac{n-1}{2}+\frac{1 / 2-1}{2}\right)^{1 / 2+2 j} \\
& \quad \cdot\left(\frac{n-1}{2}+\frac{1 / 2-1}{2}-i t\right)^{-(1 / 2+2 j)} .
\end{aligned}
$$

As such, in order to obtain a very sharp and manageable near-exact distribution for $W_{2}$, we will use, as near-exact c.f. for $W_{2}$,

$$
\begin{aligned}
& \Phi_{W_{2}}^{*}(t)=\left\{\sum_{j=0}^{m} \pi_{j}\left(\frac{n-1}{2}+\frac{1 / 2-1}{2}\right)^{1 / 2+2 j}\right. \\
& \left.\cdot\left(\frac{n-1}{2}+\frac{1 / 2-1}{2}-i t\right)^{-(1 / 2+2 j)}\right\}\left\{\prod_{j=1}^{p}\left(\frac{n-j}{2}\right)^{r_{j}}\right. \\
& \left.\cdot\left(\frac{n-j}{2}-i t\right)^{-r_{j}}\right\}=\sum_{j=0}^{m} \pi_{j}\left(\frac{n-1}{2}\right. \\
& \left.+\frac{1 / 2-1}{2}\right)^{1 / 2+2 j}\left(\frac{n-1}{2}+\frac{1 / 2-1}{2}-i t\right)^{-(1 / 2+2 j)} \\
& \cdot \prod_{j=1}^{p}\left(\frac{n-j}{2}\right)^{r_{j}}\left(\frac{n-j}{2}-i t\right)^{-r_{j}},
\end{aligned}
$$

where the weights $\pi_{j}, j=0, \ldots, m-1$, will be determined in such a way that

$$
\left.\frac{\partial^{h}}{\partial t^{h}} \Phi_{W_{2}}(t)\right|_{t=0}=\left.\frac{\partial^{h}}{\partial t^{h}} \Phi_{W_{2}}^{*}(t)\right|_{t=0}, \quad h=1, \ldots, m,
$$

with $\pi_{m}=1-\sum_{j=0}^{m-1} \pi_{j}$.

$\Phi_{W_{2}}^{*}(t)$ is the c.f. of a mixture of $m+1$ Generalized NearInteger Gamma (GNIG) distributions of depth $p+1$ (see [5] for the GNIG distribution).
As such, using the notation for the p.d.f. and c.d.f. of the GNIG distribution used in Section 3 of [6], the near-exact p.d.f.'s and c.d.f.s for $W_{2}=-\log \Lambda_{2}$ and $\Lambda_{2}$ are

$$
\begin{aligned}
& f_{W_{2}}^{*}(w)=\sum_{j=0}^{m} \pi_{j} f^{\mathrm{GNIG}}\left(w \mid r_{1}, \ldots, r_{p}, \frac{1}{2}+2 j ; \frac{n-1}{2},\right. \\
& \left.\ldots, \frac{n-p}{2}, \frac{n-1}{2}+\frac{1 / 2-1}{2} ; p+1\right), \\
& F_{W_{2}}^{*}(w)=\sum_{j=0}^{m} \pi_{j} F^{\mathrm{GNIG}}\left(w \mid r_{1}, \ldots, r_{p}, \frac{1}{2}+2 j ; \frac{n-1}{2},\right. \\
& \left.\ldots, \frac{n-p}{2}, \frac{n-1}{2}+\frac{1 / 2-1}{2} ; p+1\right), \\
& f_{\Lambda_{2}}^{*}(\ell)=\sum_{j=0}^{m} \pi_{j} f^{\mathrm{GNIG}}\left(-\log \ell \mid r_{1}, \ldots, r_{p}, \frac{1}{2}+2 j ;\right. \\
& \left.\frac{n-1}{2}, \ldots, \frac{n-p}{2}, \frac{n-1}{2}+\frac{1 / 2-1}{2} ; p+1\right) \frac{1}{\ell}, \\
& F_{\Lambda_{2}}^{*}(\ell)=\sum_{j=0}^{m} \pi_{j}\left(1-F^{\mathrm{GNIG}}\left(-\log \ell \mid r_{1}, \ldots, r_{p}, \frac{1}{2}\right.\right. \\
& \left.\left.+2 j ; \frac{n-1}{2}, \ldots, \frac{n-p}{2}, \frac{n-1}{2}+\frac{1 / 2-1}{2} ; p+1\right)\right),
\end{aligned}
$$

with $r_{1}, \ldots, r_{p}$ given by (15).

In Table 2 we may analyze values of the measure $\Delta$ in (5) for the near-exact distributions developed in this section, for different values of $p$ and different sample sizes. We may see how these near-exact distributions display very low values of the measure $\Delta$, indicating an extremely good proximity to the exact distribution, even for very small sample sizes, and how they display a sharp asymptotic behavior for increasing values of $p$ and $n$.

In Table 3 we may analyze the asymptotic quantiles for $n W_{2}$ for the common chi-square asymptotic approximation for l.r. statistics, here with $p(p+3) / 2-\lfloor(p+2) / 2\rfloor$ degrees of freedom and the quantiles for the near-exact distributions that equate 2, 6, and 10 exact moments. These quantiles are shown with 26 decimal places in order to make it possible to identify the number of correct decimal places for the quantiles of the near-exact distributions that match 2 and 6 exact moments. We should note that the quantiles of the nearexact distributions that match 10 exact moments always have much more than 26 decimal places that are correct. Also for the statistic in this section, we may see the lack of precision of the asymptotic chi-square quantiles.

\section{The Likelihood Ratio Test for the Simultaneous Test of Equality of Means and Compound Symmetry of the Covariance Matrix}

Let us assume that $\underline{X} \sim N_{p}(\underline{\mu}, \Sigma)$, with $\underline{\mu}=\left[\mu_{1}, \ldots, \mu_{p}\right]^{\prime}$. We are interested in testing the hypothesis

$$
\begin{gathered}
H_{0}: \mu_{1}=\cdots=\mu_{p} ; \\
\Sigma=\Sigma_{c s},
\end{gathered}
$$


TABLE 2: Values of the measure $\Delta$ in (5), for the near-exact distributions of the l.r. test statistic $\Lambda_{2}$ in (17), which match $m$ exact moments, for different values of $p$ and samples of size $n=p+1,50,450$.

\begin{tabular}{|c|c|c|c|c|c|c|}
\hline \multirow{2}{*}{$p$} & \multirow{2}{*}{$n$} & \multicolumn{5}{|c|}{$m$} \\
\hline & & 2 & 4 & 6 & 10 & 20 \\
\hline \multirow[t]{3}{*}{10} & 11 & $2.12 \times 10^{-10}$ & $5.95 \times 10^{-14}$ & $1.23 \times 10^{-16}$ & $1.35 \times 10^{-20}$ & $1.02 \times 10^{-26}$ \\
\hline & 60 & $3.12 \times 10^{-14}$ & $2.32 \times 10^{-20}$ & $1.46 \times 10^{-25}$ & $2.80 \times 10^{-34}$ & $1.41 \times 10^{-50}$ \\
\hline & 460 & $1.52 \times 10^{-19}$ & $3.22 \times 10^{-29}$ & $5.78 \times 10^{-38}$ & $9.53 \times 10^{-54}$ & $1.70 \times 10^{-87}$ \\
\hline \multirow[t]{3}{*}{15} & 16 & $4.12 \times 10^{-12}$ & $9.90 \times 10^{-17}$ & $2.09 \times 10^{-20}$ & $4.06 \times 10^{-26}$ & $1.32 \times 10^{-35}$ \\
\hline & 65 & $5.95 \times 10^{-15}$ & $1.60 \times 10^{-21}$ & $3.86 \times 10^{-27}$ & $1.28 \times 10^{-36}$ & $1.59 \times 10^{-54}$ \\
\hline & 465 & $4.76 \times 10^{-20}$ & $5.11 \times 10^{-30}$ & $4.92 \times 10^{-39}$ & $2.69 \times 10^{-55}$ & $5.39 \times 10^{-90}$ \\
\hline \multirow[t]{3}{*}{25} & 26 & $3.00 \times 10^{-14}$ & $2.91 \times 10^{-20}$ & $2.75 \times 10^{-25}$ & $1.56 \times 10^{-33}$ & $1.83 \times 10^{-48}$ \\
\hline & 75 & $5.13 \times 10^{-16}$ & $2.85 \times 10^{-23}$ & $1.47 \times 10^{-29}$ & $2.52 \times 10^{-40}$ & $3.53 \times 10^{-61}$ \\
\hline & 475 & $9.86 \times 10^{-21}$ & $3.91 \times 10^{-31}$ & $1.45 \times 10^{-40}$ & $1.30 \times 10^{-57}$ & $4.82 \times 10^{-94}$ \\
\hline \multirow[t]{3}{*}{50} & 51 & $4.11 \times 10^{-17}$ & $4.95 \times 10^{-25}$ & $6.07 \times 10^{-32}$ & $6.98 \times 10^{-44}$ & $2.02 \times 10^{-67}$ \\
\hline & 100 & $9.04 \times 10^{-18}$ & $3.48 \times 10^{-26}$ & $1.27 \times 10^{-33}$ & $1.16 \times 10^{-46}$ & $5.22 \times 10^{-73}$ \\
\hline & 500 & $9.25 \times 10^{-22}$ & $7.77 \times 10^{-33}$ & $6.23 \times 10^{-43}$ & $2.77 \times 10^{-61}$ & $7.62 \times 10^{-101}$ \\
\hline
\end{tabular}

where $\Sigma_{c s}$ represents a compound symmetric matrix, as defined in (3).

For a sample of size $n$, the $(2 / n)$ th power of the l.r. test statistic is (see [2])

$$
\Lambda_{3}=\frac{|A|}{(\widehat{a}+(p-1) \hat{b})\left(\widehat{a}-\widehat{b}+S^{* 2}\right)^{p-1}},
$$

where

$$
A=\left[a_{j k}\right]=X^{\prime}\left(I_{n}-\frac{1}{n} E_{n n}\right) X,
$$

with $X$ being the $n \times p$ sample matrix and $E_{n n}$ a matrix of 1's of dimension $n \times n$,

$$
\begin{aligned}
\widehat{a} & =\frac{1}{p} \sum_{j=1}^{p} a_{j j}, \\
\widehat{b} & =\frac{2}{p(p-1)} \sum_{j=1}^{p-1} \sum_{k=j+1}^{p} a_{j k}, \\
S^{* 2} & =\frac{1}{p-1} \sum_{j=1}^{p}\left(\bar{X}_{j}-\bar{X}\right)^{2},
\end{aligned}
$$

with

$$
\begin{gathered}
\bar{X}_{j}=\frac{1}{n} \sum_{i=1}^{n} X_{j i}, \\
\bar{X}=\frac{1}{p} \sum_{j=1}^{p} \bar{X}_{j} .
\end{gathered}
$$

Wilks [2] has also shown that

$$
\Lambda_{3} \stackrel{d}{\equiv} \prod_{j=2}^{p} Y_{j}
$$

where

$$
Y_{j} \sim \operatorname{Beta}\left(\frac{n-j}{2}, \frac{j-2}{p-1}+\frac{j}{2}\right),
$$

form a set of $p-1$ independent r.v.s.

As such, the $h$ th moment of $\Lambda_{3}$ may be written as

$$
\begin{array}{r}
E\left(\Lambda_{3}^{h}\right) \\
=\prod_{j=2}^{p} \frac{\Gamma(n / 2+(j-2) /(p-1)) \Gamma((n-j) / 2+h)}{\Gamma((n-j) / 2) \Gamma(n / 2+(j-2) /(p-1)+h)}, \\
\left(h>-\frac{n-p}{2}\right) .
\end{array}
$$

Since the expression in (33) remains valid for any complex $h$, we may write the c.f. of $W_{3}=-\log \Lambda_{3}$ as

$$
\begin{aligned}
\Phi_{W_{3}}(t) & =E\left(\Lambda_{3}^{-i t}\right) \\
= & \prod_{j=2}^{p} \frac{\Gamma(n / 2+(j-2) /(p-1)) \Gamma((n-j) / 2-i t)}{\Gamma((n-j) / 2) \Gamma(n / 2+(j-2) /(p-1)-i t)},
\end{aligned}
$$

which may be rewritten as

$$
\begin{aligned}
& \Phi_{W_{3}}(t) \\
& =\{\underbrace{\left\{\prod_{j=2}^{p} \frac{\Gamma(n / 2+(j-2) /(p-1)) \Gamma((n-j) / 2+\lfloor(j-2) /(p-1)+j / 2\rfloor-i t)}{\Gamma((n-j) / 2+\lfloor(j-2) /(p-1)+j / 2\rfloor) \Gamma(n / 2+(j-2) /(p-1)-i t)}\right\}}_{\Phi_{W_{3}, 1}(t)} \underbrace{\left\{\prod_{j=2}^{p} \frac{\Gamma((n-j) / 2+\lfloor(j-2) /(p-1)+j / 2\rfloor) \Gamma((n-j) / 2-i t)}{\Gamma((n-j) / 2) \Gamma((n-j) / 2+\lfloor(j-2) /(p-1)+j / 2\rfloor-i t)}\right\}}_{\Phi_{W_{3}, 2}(t)} .(.)
\end{aligned}
$$




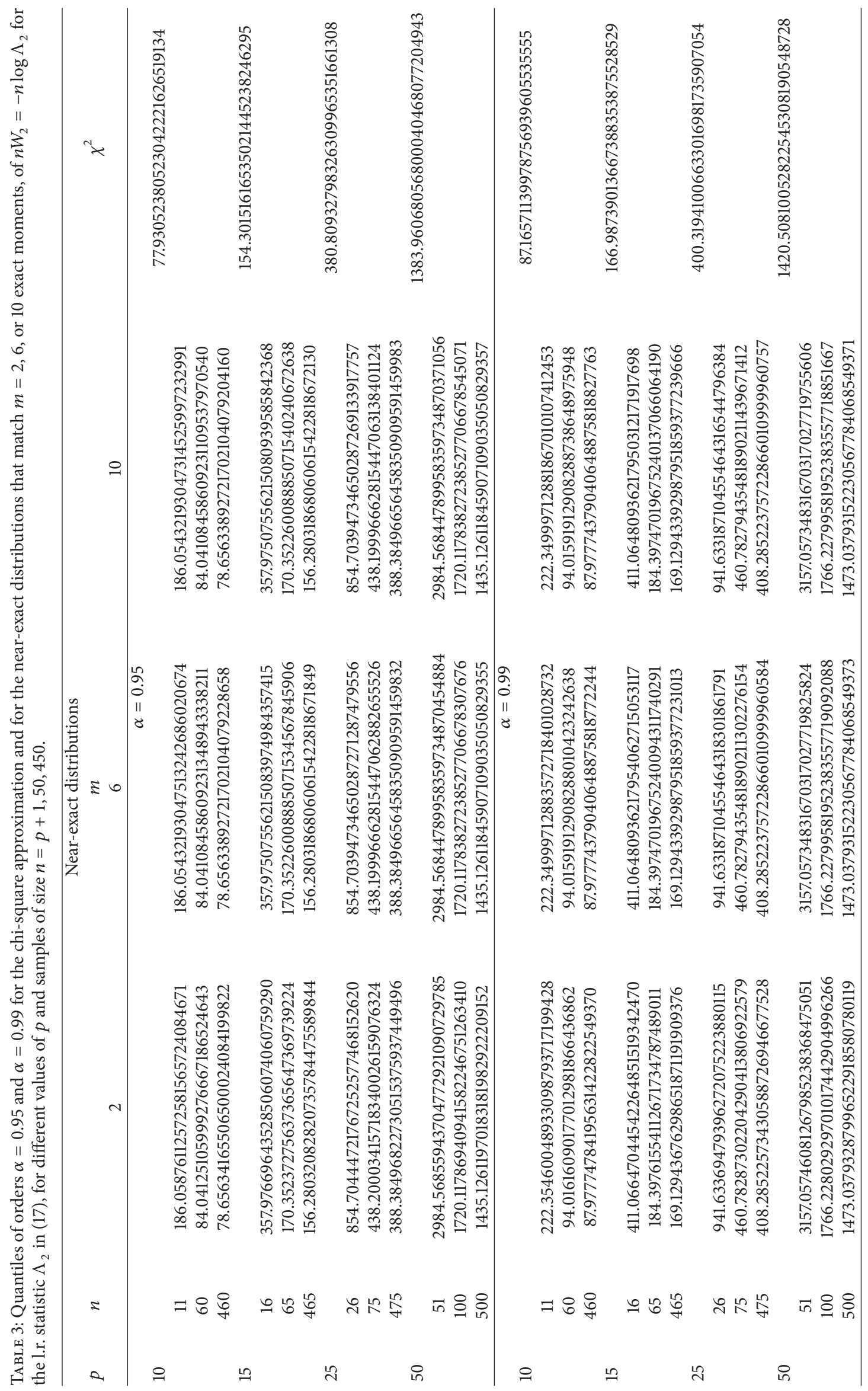


Then, we may apply on $\Phi_{W_{3}, 2}(t)$ the relation

to obtain

$$
\frac{\Gamma(a+n)}{\Gamma(a)}=\prod_{\ell=0}^{n-1}(a+\ell), \quad \forall a \in \mathbb{C}, n \in \mathbb{N}
$$

$$
\begin{aligned}
& \Phi_{W_{3}}(t)=\left\{\prod_{j=3}^{p} \frac{\Gamma(n / 2+(j-2) /(p-1)) \Gamma((n-j) / 2+\lfloor(j-2) /(p-1)+j / 2\rfloor-i t)}{\Gamma((n-j) / 2+\lfloor(j-2) /(p-1)+j / 2\rfloor) \Gamma(n / 2+(j-2) /(p-1)-i t)}\right\} \\
& \left\{\prod_{j=2}^{p} \prod_{\ell=0}^{\lfloor(j-2) /(p-1)+j / 2\rfloor-1}\left(\frac{n-j}{2}+\ell\right)\left(\frac{n-j}{2}+\ell-i t\right)^{-1}\right\} \\
& =\underbrace{\left\{\prod_{j=3}^{p} \frac{\Gamma(n / 2+(j-2) /(p-1)) \Gamma((n-j) / 2+\lfloor(j-2) /(p-1)+j / 2\rfloor-i t)}{\Gamma((n-j) / 2+\lfloor(j-2) /(p-1)+j / 2\rfloor) \Gamma(n / 2+(j-2) /(p-1)-i t)}\right\}}_{\Phi_{W_{3}, 1}(t)} \underbrace{\left\{\prod_{j=1}^{p}\left(\frac{n-j}{2}\right)^{r_{j}}\left(\frac{n-j}{2}-i t\right)^{-r_{j}}\right\}}_{\Phi_{W_{3}, 2}(t)},
\end{aligned}
$$

with

$$
r_{j}= \begin{cases}\frac{p}{4}-1, & j=1, \text { if }(p \bmod 4)=0, \\ \left\lfloor\frac{(p+1)}{4}\right\rfloor & j=1, \text { if }(p \bmod 4) \neq 0, \\ \left\lfloor\frac{(p-j+2)}{2}\right\rfloor & j=2, \ldots, p .\end{cases}
$$

Expression (37) shows that the exact distribution of $W_{3}$ is the same as that of the sum of GIG distributed r.v.s of depth $p$ with an independent sum of $p-2$ independent Logbeta $((n-$ $j) / 2+\lfloor(j-2) /(p-1)+j / 2\rfloor,(j-2) /(p-1)+j / 2-\lfloor(j-$ $2) /(p-1)+j / 2\rfloor)$ r.v.s.

Our aim in building the near-exact distribution will be to keep $\Phi_{W_{3}, 2}(t)$ unchanged and approximate asymptotically $\Phi_{W_{3}, 1}(t)$.

In order to obtain this asymptotic approximation, we will need to use a different approach from the one used in the previous section. We will use the result in sec. 5 of [9], which implies that a $\operatorname{Logbeta}(a, b)$ distribution may be asymptotically replaced by an infinite mixture of $\Gamma(b+j, a)(j=0,1, \ldots)$ distributions.

Using a somewhat heuristic approach, we will thus approximate $\Phi_{W_{3}, 1}(t)$ by a mixture of $\Gamma\left(r+j, \lambda^{*}\right)$ distributions where

$$
\begin{aligned}
r & =\sum_{j=2}^{p} \frac{j-2}{p-1}+\frac{j}{2}-\left\lfloor\frac{j-2}{p-1}+\frac{j}{2}\right\rfloor \\
& =\frac{p-3}{2}+\frac{((p+1) \bmod 2)+((p+1) \bmod 4)}{2^{(p+1) \bmod 4}}
\end{aligned}
$$

is the sum of the second parameters of the Logbeta r.v.s in $\Phi_{W_{3}, 1}(t)$ and $\lambda^{*}$ is the common rate parameter in the mixture of two Gamma distributions that matches the first 4 moments of $\Phi_{W_{3}, 1}(t)$, that is, $\lambda^{*}$ in

$$
\begin{aligned}
& \frac{d^{h}}{d t^{h}}\left(p\left(\lambda^{*}\right)^{r_{1}}\left(\lambda^{*}-i t\right)^{-r_{1}}\right. \\
& \left.+(1-p)\left(\lambda^{*}\right)^{r_{2}}\left(\lambda^{*}-i t\right)^{-r_{2}}\right)\left.\right|_{t=0}=\frac{d^{h}}{d t^{h}} \\
& \left.\cdot \Phi_{W_{3}, 1}(t)\right|_{t=0}, \quad h=1, \ldots, 4 .
\end{aligned}
$$

As such, in order to build the near-exact distributions for $W_{3}$, we will use, as near exact c.f. for $W_{3}$,

$$
\begin{gathered}
\Phi_{W_{3}}^{*}(t)=\left\{\sum_{j=0}^{m} \pi_{j}\left(\lambda^{*}\right)^{r+j}\left(\lambda^{*}-i t\right)^{-(r+j)}\right\} \\
\cdot\left\{\prod_{j=1}^{p}\left(\frac{n-j}{2}\right)^{r_{j}}\left(\frac{n-j}{2}-i t\right)^{-r_{j}}\right\} \\
=\sum_{j=0}^{m} \pi_{j}\left(\lambda^{*}\right)^{r+j}\left(\lambda^{*}-i t\right)^{-(r+j)} \\
\cdot \prod_{j=1}^{p}\left(\frac{n-j}{2}\right)^{r_{j}}\left(\frac{n-j}{2}-i t\right)^{-r_{j}},
\end{gathered}
$$

where the weights $\pi_{j}, j=0, \ldots, m-1$, will be determined in such a way that

$$
\left.\frac{\partial^{h}}{\partial t^{h}} \Phi_{W_{3}}(t)\right|_{t=0}=\left.\frac{\partial^{h}}{\partial t^{h}} \Phi_{W_{3}}^{*}(t)\right|_{t=0}, \quad h=1, \ldots, m,
$$

with $\pi_{m}=1-\sum_{j=0}^{m-1} \pi_{j}$.

The c.f. in (41) is, for integer $r$, the c.f. of a mixture of $m+$ 1 GIG distributions of depth $p+1$ or, for noninteger $r$, the 
TABLE 4: Values of the measure $\Delta$ in (5), for the near-exact distributions of the 1.r. test statistic $\Lambda_{3}$ in (26), which match $m$ exact moments, for different values of $p$ and samples of size $n=p+1,50,450$.

\begin{tabular}{ccccccc}
\hline$p$ & $n$ & 2 & 4 & $m$ & 6 & 10 \\
\hline 10 & 11 & $9.68 \times 10^{-9}$ & $3.84 \times 10^{-13}$ & $1.07 \times 10^{-15}$ & $1.36 \times 10^{-20}$ & $5.71 \times 10^{-29}$ \\
& 60 & $3.01 \times 10^{-10}$ & $1.24 \times 10^{-15}$ & $3.49 \times 10^{-19}$ & $6.90 \times 10^{-26}$ & $8.11 \times 10^{-40}$ \\
& 460 & $6.80 \times 10^{-13}$ & $3.93 \times 10^{-20}$ & $1.95 \times 10^{-25}$ & $9.75 \times 10^{-36}$ & $7.94 \times 10^{-58}$ \\
\hline 15 & 16 & $9.99 \times 10^{-10}$ & $1.62 \times 10^{-14}$ & $4.49 \times 10^{-19}$ & $2.91 \times 10^{-27}$ & $3.19 \times 10^{-41}$ \\
& 65 & $9.85 \times 10^{-11}$ & $3.36 \times 10^{-16}$ & $2.24 \times 10^{-21}$ & $9.91 \times 10^{-31}$ & $1.76 \times 10^{-49}$ \\
& 465 & $2.81 \times 10^{-13}$ & $2.09 \times 10^{-20}$ & $3.35 \times 10^{-27}$ & $9.96 \times 10^{-40}$ & $3.22 \times 10^{-62}$ \\
\hline 25 & 26 & $6.12 \times 10^{-11}$ & $1.73 \times 10^{-16}$ & $6.85 \times 10^{-22}$ & $1.67 \times 10^{-32}$ & $1.52 \times 10^{-50}$ \\
& 75 & $1.87 \times 10^{-11}$ & $2.86 \times 10^{-17}$ & $5.76 \times 10^{-23}$ & $1.12 \times 10^{-33}$ & $2.66 \times 10^{-55}$ \\
& 475 & $7.06 \times 10^{-14}$ & $3.38 \times 10^{-21}$ & $2.21 \times 10^{-28}$ & $6.35 \times 10^{-42}$ & $2.53 \times 10^{-65}$ \\
\hline 50 & 51 & $1.78 \times 10^{-12}$ & $5.44 \times 10^{-19}$ & $2.24 \times 10^{-25}$ & $9.80 \times 10^{-37}$ & $8.74 \times 10^{-61}$ \\
& 100 & $2.35 \times 10^{-12}$ & $9.83 \times 10^{-19}$ & $4.94 \times 10^{-25}$ & $2.44 \times 10^{-36}$ & $1.64 \times 10^{-58}$ \\
& 500 & $2.25 \times 10^{-14}$ & $5.94 \times 10^{-22}$ & $1.76 \times 10^{-29}$ & $2.05 \times 10^{-43}$ & $8.50 \times 10^{-72}$ \\
\hline
\end{tabular}

c.f. of a mixture of $m+1$ GNIG distributions of depth $p+1$, with shape parameters $r_{1}, \ldots, r_{p}, r+j(j=0, \ldots, m)$ and rate parameters $(n-1) / 2, \ldots,(n-p) / 2, \lambda^{*}$.

This will yield, for noninteger $r$, near-exact distributions whose p.d.f.s and c.d.f.s for $W_{3}=-\log \Lambda_{3}$ and $\Lambda_{3}$ are

$$
\begin{aligned}
& f_{W_{3}}^{*}(w)=\sum_{j=0}^{m} \pi_{j} f^{\mathrm{GNIG}}\left(w \mid r_{1}, \ldots, r_{p}, r+j ; \frac{n-1}{2}, \ldots,\right. \\
& \left.\quad \frac{n-p}{2}, \lambda^{*} ; p+1\right), \\
& F_{W_{3}}^{*}(w)=\sum_{j=0}^{m} \pi_{j} F^{\mathrm{GNIG}}\left(w \mid r_{1}, \ldots, r_{p}, r+j ; \frac{n-1}{2}, \ldots,\right. \\
& \left.\quad \frac{n-p}{2}, \lambda^{*} ; p+1\right), \\
& f_{\Lambda_{3}}^{*}(\ell)=\sum_{j=0}^{m} \pi_{j} f^{\mathrm{GNIG}}\left(-\log \ell \mid r_{1}, \ldots, r_{p}, r+j ;\right. \\
& \left.\quad \frac{n-1}{2}, \ldots, \frac{n-p}{2}, \lambda^{*} ; p+1\right) \frac{1}{\ell}, \\
& \left.\left.\quad+j ; \frac{n-1}{2}, \ldots, \frac{n-p}{2}, \lambda^{*} ; p+1\right)\right),
\end{aligned}
$$

with $r_{1}, \ldots, r_{p}$ given by (38). For integer $r$, we will only have to replace in the above expressions the GNIG p.d.f. and c.d.f. by the GIG p.d.f. and c.d.f., respectively.

In Table 4 , we may analyze values of the measure $\Delta$ in (5) for the near-exact distributions developed in this section, for different values of $p$ and different sample sizes. We may see how these near-exact distributions display, once again, very low values of the measure $\Delta$ even for very small sample sizes, indicating an extremely good proximity to the exact distribution and how, once again, they display a sharp asymptotic behavior for increasing values of $p$ and $n$, although for large values of $p$, namely, for $p=50$ in Table 4 , one may have to consider larger values of $n$ in order to be able to observe the asymptotic behavior in terms of sample size.

The asymptotic quantiles for $n W_{3}$ in Table 5, for the common chi-square asymptotic approximation for l.r. statistics, now with $p(p+3) / 2-3$ degrees of freedom, display again, as in the previous sections, an almost shocking lack of precision, mainly for small sample sizes and/or larger numbers of variables. On the other hand, the near-exact quantiles show a steady evolution towards the exact quantiles for increasing number of exact moments matched, with the quantiles for the near-exact distributions that match 6 exact moments displaying more than 20 correct decimal places, for the larger sample sizes.

\section{The Likelihood Ratio Test for the Simultaneous Test of Nullity of Means and Compound Symmetry of the Covariance Matrix}

Let us assume now that $\underline{X} \sim N_{p}(\underline{\mu}, \Sigma)$. We are interested in testing the hypothesis

$$
\begin{aligned}
H_{0}: \underline{\mu} & =\underline{0} ; \\
\Sigma & =\Sigma_{c s} .
\end{aligned}
$$

We may write

$$
H_{0} \equiv H_{02 \mid 01} \circ H_{01}
$$

where

$$
\begin{aligned}
H_{02 \mid 01}: \mu & =\underline{0}, \text { assuming } \Sigma=\Sigma_{c s}, \\
H_{01} & : \Sigma=\Sigma_{c s} .
\end{aligned}
$$

While, for a sample of size $n$, the $(2 / n)$ th power of the l.r. statistic to test $H_{02 \mid 01}$ may be shown to be (see Appendix A for details)

$$
\Lambda_{2 \mid 1}=\frac{(\widehat{a}-\widehat{b})^{p-1}(\widehat{a}+(p-1) \widehat{b})}{\left(\widehat{a}_{0}-\widehat{b}_{0}\right)^{p-1}\left(\widehat{a}_{0}+(p-1) \hat{b}_{0}\right)},
$$




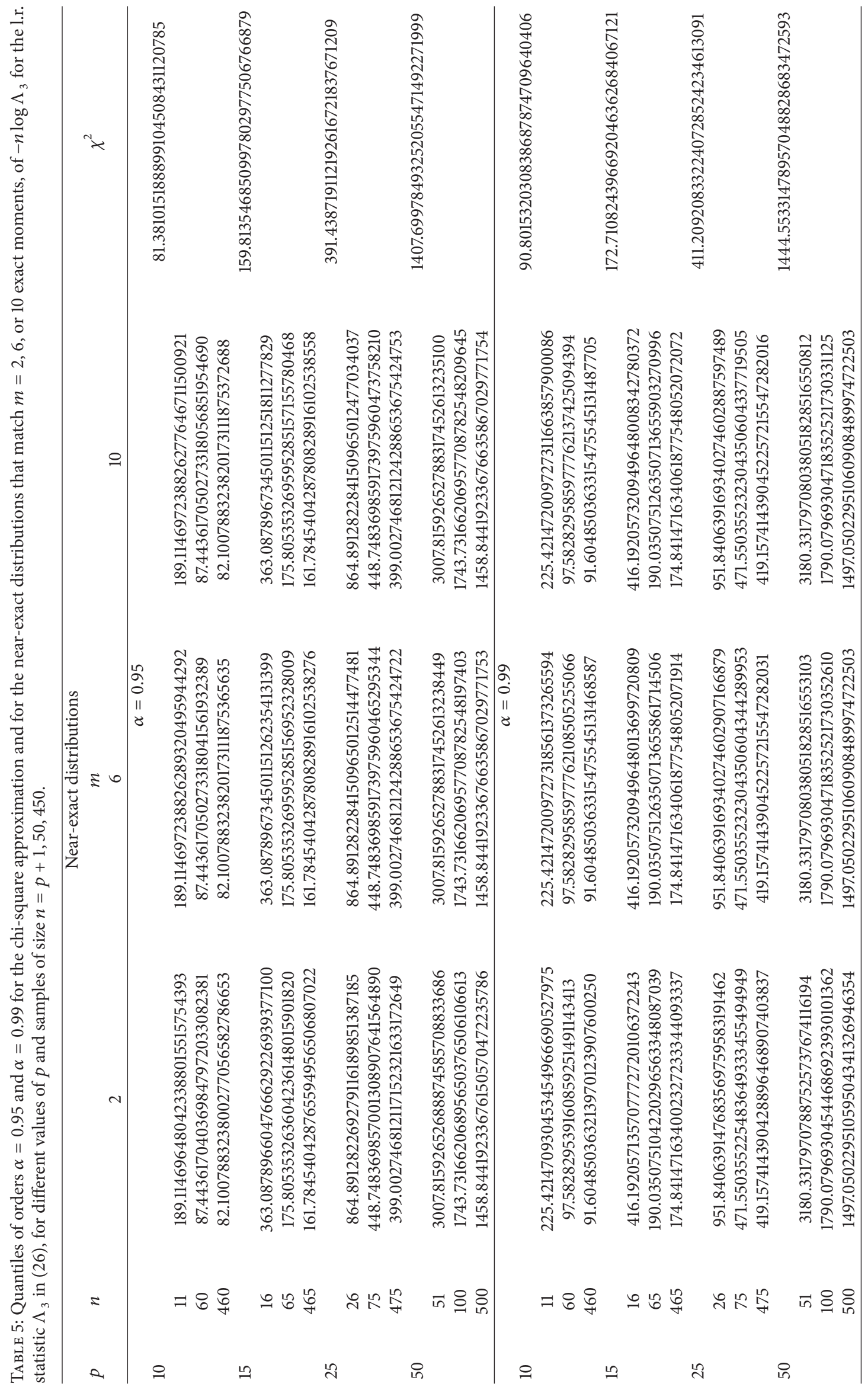


where $\widehat{a}$ and $\hat{b}$ are given by (28) and

$$
\begin{aligned}
& \widehat{a}_{0}=\frac{1}{p} \sum_{j=1}^{p} a_{0(j j)}, \\
& \widehat{b}_{0}=\frac{2}{p(p-1)} \sum_{j=1}^{p-1} \sum_{k=j+1}^{p} a_{0(j k)},
\end{aligned}
$$

with

$$
A_{0}=\left[a_{0(j k)}\right]=X^{\prime} X
$$

where $X$ is the $n \times p$ sample matrix, the l.r. test statistic to test $H_{01}$ is shown by [2] to be

$$
\Lambda_{1}=\frac{|A|}{(\widehat{a}-\widehat{b})^{p-1}(\widehat{a}+(p-1) \hat{b})},
$$

again with $\widehat{a}$ and $\widehat{b}$ given by (28) and $A$ given by (27).

The l.r. test to test $H_{0}$ in (44) is thus

$$
\Lambda_{4}=\Lambda_{1} \Lambda_{2 \mid 1}=\frac{|A|}{\left(\widehat{a}_{0}-\widehat{b}_{0}\right)^{p-1}\left(\widehat{a}_{0}+(p-1) \hat{b}_{0}\right)} .
$$

For a sample of size $n, \Lambda_{2 \mid 1}$, the $(2 / n)$ th power of the 1.r. test statistic to test $H_{02 \mid 01}$, may be shown to be distributed as (see [3] and Appendix A for details) $Y_{1}^{p-1} Y_{2}$, where $Y_{1}$ and $Y_{2}$ are independent, with

$$
\begin{aligned}
& Y_{1} \sim \operatorname{Beta}\left(\frac{(n-1)(p-1)}{2}, \frac{p-1}{2}\right), \\
& Y_{2} \sim \operatorname{Beta}\left(\frac{n-1}{2}, \frac{1}{2}\right),
\end{aligned}
$$

while [2] shows that the $(2 / n)$ th power of the l.r. statistic to test $H_{01}$ is distributed as $\prod_{j=2}^{p} Y_{j}^{*}$, where $Y_{j}^{*}$ are independent, with

$$
Y_{j}^{*} \sim \operatorname{Beta}\left(\frac{n-j}{2}, \frac{j-2}{p-1}+\frac{j-1}{2}\right) .
$$

Based on Theorem 5 in [10], it is then possible to show that the l.r. statistics to test $H_{01}$ and $H_{02 \mid 01}$ are independent, since $\Lambda_{1}$ is independent of $(\widehat{a}-\widehat{b})^{p-1}(\widehat{a}+(p-1) \widehat{b})$ and $\Lambda_{2 \mid 1}$ is built only on this statistic, since $\left(\widehat{a}_{0}-\widehat{b}_{0}\right)^{p-1}\left(\widehat{a}_{0}+(p-1) \widehat{b}_{0}\right)$ is the same statistic in a constrained subspace.

From this fact, we may show that the $(2 / n)$ th power of the l.r. statistic to test $H_{0}$ in (44), $\Lambda_{4}$, is distributed as (see Appendix B for details)

$$
\left\{\prod_{j=2}^{p} Y_{j}^{* *}\right\} Y
$$

where all r.v.s are independent, with

$$
\begin{aligned}
Y_{j}^{* *} & \sim \operatorname{Beta}\left(\frac{n-j}{2}, \frac{j-2}{p-1}+\frac{j}{2}\right), \\
Y & \sim \operatorname{Beta}\left(\frac{n-1}{2}, \frac{1}{2}\right) .
\end{aligned}
$$

We note that the r.v.s $Y_{j}^{* *}$ are the same as the r.v.s $Y_{j}$ in (31) and (32).

As such, the c.f. of $W_{4}=-\log \Lambda_{4}$ may be written as

$$
\begin{aligned}
& \Phi_{W_{4}}(t)
\end{aligned}
$$

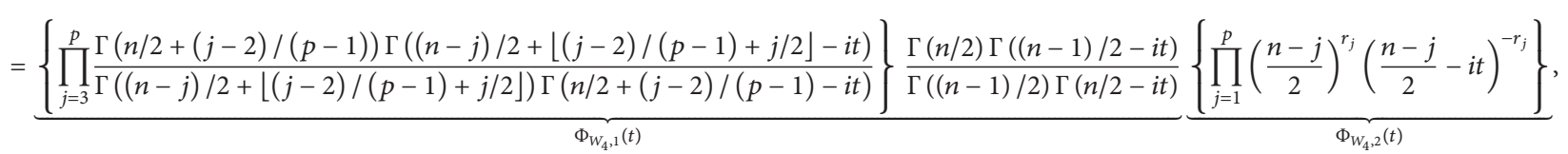

with $r_{j}$ given by (38).

Then, following a similar approach to the one used for $W_{3}$ and $\Lambda_{3}$, in the previous section, we obtain near-exact distributions with a similar structure to those in that section, now with

$$
\begin{aligned}
r & =\frac{1}{2}+\sum_{j=2}^{p} \frac{j-2}{p-1}+\frac{j}{2}-\left\lfloor\frac{j-2}{p-1}+\frac{j}{2}\right\rfloor \\
& =\frac{p-2}{2}+\frac{((p+1) \bmod 2)+((p+1) \bmod 4)}{2^{(p+1) \bmod 4}}
\end{aligned}
$$

and with $\lambda^{*}$ determined as the solution of a system of equations similar to the one in (40), with $\Phi_{W_{3}, 1}$ replaced by $\Phi_{W_{4}, 1}$.
This will yield for $\Lambda_{4}$ and $W_{4}$ near-exact distributions with p.d.f.'s and c.d.f.s given by (43), now with $r$ given by (57).

We should note that as it happens with $\Lambda_{3}$ and $W_{3}$, also for $W_{4}$ and $\Lambda_{4}, r$ may be either an integer or a half-integer, so that, in those cases where $r$ is an integer, the near-exact distributions are mixtures of GIG distributions, while when $r$ is noninteger, they are mixtures of GNIG distributions.

In Table 6 we may analyze values of the measure $\Delta$ in (5) for the near-exact distributions developed in this section, for different values of $p$ and different sample sizes. We may see how these near-exact distributions display similar properties to those of the near-exact distributions developed for $\Lambda_{3}$ in the previous section. 
TABLE 6: Values of the measure $\Delta$ in (5), for the near-exact distributions of the l.r. test statistic $\Lambda_{4}$ in (51), which match $m$ exact moments, for different values of $p$ and samples of size $n=p+1,50,450$.

\begin{tabular}{|c|c|c|c|c|c|c|}
\hline \multirow{2}{*}{$p$} & \multirow{2}{*}{$n$} & \multicolumn{5}{|c|}{$m$} \\
\hline & & 2 & 4 & 6 & 10 & 20 \\
\hline \multirow[t]{3}{*}{10} & 11 & $2.54 \times 10^{-9}$ & $5.91 \times 10^{-13}$ & $9.61 \times 10^{-16}$ & $2.01 \times 10^{-20}$ & $8.06 \times 10^{-29}$ \\
\hline & 60 & $2.80 \times 10^{-10}$ & $3.92 \times 10^{-16}$ & $4.36 \times 10^{-19}$ & $7.29 \times 10^{-26}$ & $1.73 \times 10^{-40}$ \\
\hline & 460 & $7.70 \times 10^{-13}$ & $1.42 \times 10^{-20}$ & $2.88 \times 10^{-25}$ & $1.38 \times 10^{-35}$ & $9.04 \times 10^{-58}$ \\
\hline \multirow[t]{3}{*}{15} & 16 & $1.36 \times 10^{-10}$ & $1.88 \times 10^{-14}$ & $5.28 \times 10^{-18}$ & $3.92 \times 10^{-25}$ & $9.44 \times 10^{-36}$ \\
\hline & 65 & $4.44 \times 10^{-11}$ & $1.52 \times 10^{-16}$ & $2.70 \times 10^{-20}$ & $9.22 \times 10^{-28}$ & $2.19 \times 10^{-43}$ \\
\hline & 465 & $2.05 \times 10^{-13}$ & $8.43 \times 10^{-23}$ & $3.28 \times 10^{-26}$ & $4.38 \times 10^{-37}$ & $1.62 \times 10^{-60}$ \\
\hline \multirow[t]{3}{*}{25} & 26 & $3.13 \times 10^{-11}$ & $1.07 \times 10^{-16}$ & $2.08 \times 10^{-21}$ & $2.25 \times 10^{-29}$ & $1.37 \times 10^{-45}$ \\
\hline & 75 & $7.48 \times 10^{-13}$ & $1.25 \times 10^{-18}$ & $2.38 \times 10^{-22}$ & $6.06 \times 10^{-31}$ & $5.83 \times 10^{-49}$ \\
\hline & 475 & $3.30 \times 10^{-14}$ & $1.93 \times 10^{-21}$ & $1.03 \times 10^{-27}$ & $1.73 \times 10^{-39}$ & $9.08 \times 10^{-65}$ \\
\hline \multirow[t]{3}{*}{50} & 51 & $1.49 \times 10^{-12}$ & $4.43 \times 10^{-19}$ & $1.32 \times 10^{-25}$ & $9.24 \times 10^{-37}$ & $2.37 \times 10^{-60}$ \\
\hline & 100 & $1.71 \times 10^{-12}$ & $6.92 \times 10^{-19}$ & $2.41 \times 10^{-25}$ & $2.36 \times 10^{-36}$ & $1.62 \times 10^{-58}$ \\
\hline & 500 & $1.24 \times 10^{-14}$ & $3.26 \times 10^{-22}$ & $6.45 \times 10^{-30}$ & $2.06 \times 10^{-43}$ & $1.20 \times 10^{-70}$ \\
\hline
\end{tabular}

In Table 7, the asymptotic chi-square quantiles are made available for the common chi-square asymptotic approximation for l.r. statistics, now with $p(p+3) / 2-2$ degrees of freedom, as well as the near-exact quantiles for $n W_{4}$. Similar conclusions to those drawn in the previous sections apply here.

\section{The Likelihood Ratio Test for the Simultaneous Test of Equality of Means and Sphericity of the Covariance Matrix}

If $\underline{X} \sim N_{p}(\underline{\mu}, \Sigma)$, where $\underline{\mu}=\left[\mu_{1}, \ldots, \mu_{p}\right]^{\prime}$, and we are interested in testing the null hypothesis

$$
\begin{aligned}
H_{0}: \mu_{1} & =\cdots=\mu_{p} ; \\
\Sigma & =\sigma^{2} I_{p} \quad\left(\text { with } \sigma^{2} \text { unspecified }\right),
\end{aligned}
$$

we may write

$$
H_{0} \equiv H_{02 \mid 01} \circ H_{01}
$$

where

$$
\begin{aligned}
H_{02 \mid 01}: \mu_{1} & =\cdots=\mu_{p}, \text { assuming } \Sigma=\sigma^{2} I_{p}, \\
H_{01}: \Sigma & =\sigma^{2} I_{p},
\end{aligned}
$$

where, for a sample of size $n$, the $(2 / n)$ th power of the l.r. statistic to test $H_{02 \mid 01}$, versus an alternative hypothesis that assumes sphericity for the covariance matrix and no structure for the mean vector, may be shown to be (see Appendix C for details)

$$
\Lambda_{2 \mid 1}=\left(\frac{\operatorname{tr}(A)}{\operatorname{tr}\left(A_{0}\right)}\right)^{p}
$$

where $A$ is the matrix in (27) and

$$
A_{0}=\left(X-E_{n 1} \underline{\widehat{\mu}}^{\prime}\right)^{\prime}\left(X-E_{n 1} \underline{\widehat{\mu}}^{\prime}\right),
$$

with

$$
\underline{\widehat{\mu}}=\frac{1}{p} E_{p 1} E_{1 p} \underline{\bar{X}}=\frac{1}{p} E_{p p} \underline{\bar{X}} .
$$

We have

$$
\begin{aligned}
\operatorname{tr}(A) & =\sum_{j=1}^{p} \sum_{i=1}^{n}\left(X_{i j}-\bar{X}_{j}\right)^{2}, \\
\operatorname{tr}\left(A_{0}\right) & =\sum_{j=1}^{p} \sum_{i=1}^{n}\left(X_{i j}-\bar{X}\right)^{2} \\
& =\underbrace{\sum_{j=1}^{p} \sum_{i=1}^{n}\left(X_{i j}-\bar{X}_{j}\right)^{2}}_{A^{*}}+\underbrace{n \sum_{j=1}^{p}\left(\bar{X}_{j}-\bar{X}\right)^{2}}_{B^{*}},
\end{aligned}
$$

where $X=\left[X_{i j}\right](i=1, \ldots, n ; j=1, \ldots, p)$ is the sample matrix and

$$
\bar{X}=\frac{1}{p} \sum_{j=1}^{p} \bar{X}_{j}, \quad \text { with } \bar{X}_{j}=\frac{1}{n} \sum_{i=1}^{n} X_{i j}
$$

In (65), from standard theory on normal r.v.'s, since $X_{i j} \sim$ $N\left(\mu_{j}, \sigma^{2}\right)$, independent for $i=1, \ldots, n$,

$$
\frac{\sum_{i=1}^{n}\left(X_{i j}-\bar{X}_{j}\right)^{2}}{\sigma^{2}} \sim \chi_{n-1}^{2}, \quad(j=1, \ldots, p),
$$




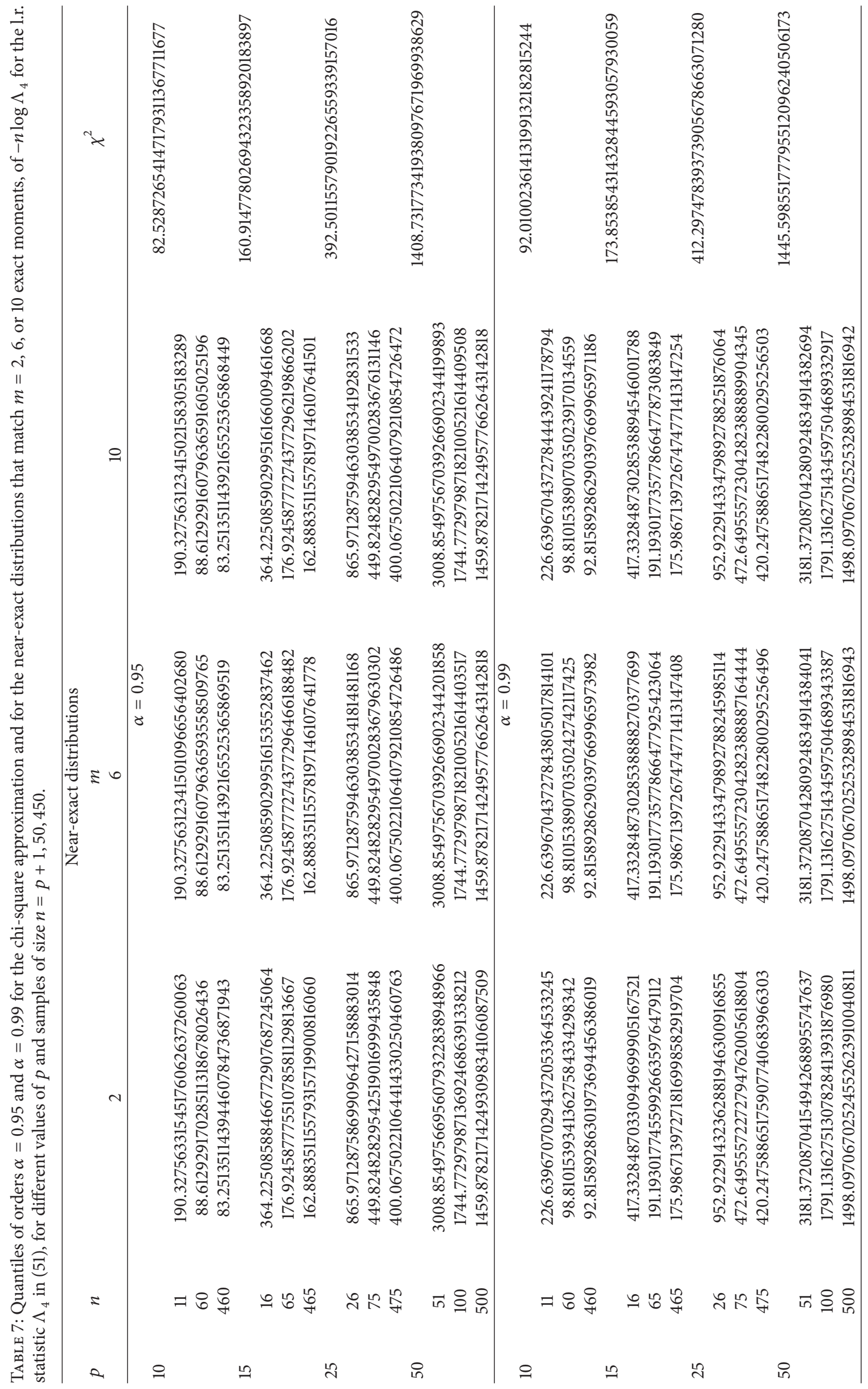


and, since, under $H_{02 \mid 01}$, we have $\bar{X}_{j} \sim N\left(\mu, \sigma^{2} / n\right)$, i.i.d. for $j=1, \ldots, p$, under this null hypothesis,

$$
\frac{\sum_{j=1}^{p}\left(\bar{X}_{j}-\bar{X}\right)^{2}}{\sigma^{2} / n} \sim \chi_{p-1}^{2} .
$$

Thus, since the r.v.s in (67) are independent for $j=1, \ldots, p$,

$$
\frac{A^{*}}{\sigma^{2}} \sim \chi_{(n-1) p}^{2}
$$

while, from (68),

$$
\frac{B^{*}}{\sigma^{2}} \sim \chi_{p-1}^{2}
$$

Since $A^{*}$ and $B^{*}$ are independent, given that $A^{*}$ is independent of all $\bar{X}_{j}(j=1, \ldots, p)$ and $B^{*}$ is defined only from the $\bar{X}_{j}$, then

$$
\begin{aligned}
\frac{\operatorname{tr}(A)}{\operatorname{tr}\left(A_{0}\right)} & =\frac{A^{*}}{A^{*}+B^{*}} \sim \operatorname{Beta}\left(\frac{(n-1) p}{2}, \frac{p-1}{2}\right), \\
\Lambda_{2 \mid 1} & \sim\left(\operatorname{Beta}\left(\frac{(n-1) p}{2}, \frac{p-1}{2}\right)\right)^{p} .
\end{aligned}
$$

From $[6,11]$ and $[12$, sec. 10.7$]$, the $(2 / n)$ th power of the l.r. statistic to test $H_{01}$ in (60) is given by

$$
\Lambda_{1}=\frac{|A|}{(\operatorname{tr}((1 / p) A))^{p}}
$$

with (see [6])

$$
\Lambda_{1} \stackrel{d}{\equiv} \prod_{j=2}^{p} Y_{j}
$$

where, for $j=2, \ldots, p$,

$$
Y_{j} \sim \operatorname{Beta}\left(\frac{n-j}{2}, \frac{j-1}{p}+\frac{j-1}{2}\right)
$$

are a set of $p-1$ independent r.v.s.

$$
\begin{aligned}
\Phi_{W_{2}}(t) & =\frac{\Gamma((n p-1) / 2) \Gamma((n p-p) / 2-p i t)}{\Gamma((n p-1) / 2-p i t) \Gamma((n p-p) / 2)} \\
& =\frac{\Gamma((n p-1) / 2) \Gamma((n p-2) / 2-p i t)}{\Gamma((n p-1) / 2-p i t) \Gamma((n p-2) / 2)} \frac{\Gamma((n p-2) / 2) \Gamma((n p-p) / 2-p i t)}{\Gamma((n p-2) / 2-p i t) \Gamma((n p-p) / 2)} \\
& =\frac{\Gamma((n p-1) / 2) \Gamma((n p-2) / 2-p i t)}{\Gamma((n p-1) / 2-p i t) \Gamma((n p-2) / 2)}\left\{\prod_{\ell=0}^{p / 2-2}\left(\frac{n p-p}{2}+\ell\right)\left(\frac{n p-p}{2}-p i t+\ell\right)^{-1}\right\} \\
& =\frac{\Gamma((n p-1) / 2) \Gamma((n p-2) / 2-p i t)}{\Gamma((n p-1) / 2-p i t) \Gamma((n p-2) / 2)}\left\{\prod_{j=0}^{p / 2-2}\left(\frac{n-1+(2 j) / p}{2}\right)\left(\frac{n-1+(2 j) / p}{2}-i t\right)^{-1}\right\} .
\end{aligned}
$$

where all r.v.s are independent, $Y_{j}$ are the r.v's in (75), and $Y^{*}$ is a r.v. with the same distribution as $\Lambda_{2 \mid 1}$ in (72).

Let us take $W_{5}=-\log \Lambda_{5}, W_{2}=-\log \Lambda_{2 \mid 1}$, and $W_{1}=$ $-\log \Lambda_{1}$. Then we will have

$$
\Phi_{W_{5}}(t)=\Phi_{W_{1}}(t) \Phi_{W_{2}}(t),
$$

where, using (36), we may write, for odd $p$,

$$
\begin{aligned}
\Phi_{W_{2}}(t)=E\left(e^{t W_{2}}\right)=E\left(e^{-t \log \Lambda_{2 \mid 1}}\right)=E\left(\Lambda_{2 \mid 1}^{-i t}\right) \\
=\frac{\Gamma((n p-1) / 2) \Gamma((n p-p) / 2-p i t)}{\Gamma((n p-1) / 2-p i t) \Gamma((n p-p) / 2)} \\
=\prod_{\ell=0}^{(p-1) / 2-1}\left(\frac{n p-p}{2}+\ell\right)\left(\frac{n p-p}{2}-p i t+\ell\right)^{-1} \\
=\prod_{j=0}^{(p-1) / 2-1}\left(\frac{n p-p+2 j}{2}\right)\left(\frac{n p-p+2 j}{2}-p i t\right)^{-1} \\
=\prod_{j=0}^{(p-1) / 2-1}\left(\frac{n-1+(2 j) / p}{2}\right) \\
\cdot\left(\frac{n-1+(2 j) / p}{2}-i t\right)^{-1},
\end{aligned}
$$

and, for even $p$, following similar steps, 
Taking for $\Phi_{W_{1}}(t)$ the expression for $\Phi_{W_{3}}(t)$ in (A.6) in [6], we may write

$$
\begin{aligned}
& \Phi_{W_{5}}(t) \\
& =\underbrace{\left\{\prod_{j=1}^{p-k^{*}} \frac{\Gamma((n-1) / 2+(j-1) / p) \Gamma((n-1) / 2-i t)}{\Gamma((n-1) / 2+(j-1) / p-i t) \Gamma((n-1) / 2)}\right\}\left\{\prod_{j=p-k^{*}+1}^{p} \frac{\Gamma((n-1) / 2+(j-1) / p) \Gamma(n / 2-i t)}{\Gamma((n-1) / 2+(j-1) / p-i t) \Gamma(n / 2)}\right\}\left(\frac{\Gamma((n p-1) / 2) \Gamma((n p-2) / 2-p i t)}{\Gamma((n p-1) / 2-p i t) \Gamma((n p-2) / 2)}\right)^{(p+1) \bmod 2}}_{\Phi_{W_{5}, 1}(t)} \\
& \underbrace{\left\{\prod_{j=1}^{p}\left(\frac{n-j}{2}\right)^{r_{j}}\left(\frac{n-j}{2}-i t\right)^{-r_{j}}\right\}\left\{\prod_{j=1}^{L(p-1) / 2\rfloor-1}\left(\frac{n-1+(2 j) / p}{2}\right)\left(\frac{n-1+(2 j) / p}{2}-i t\right)^{-1}\right\}}_{\Phi_{W_{5}, 2}(t)}
\end{aligned}
$$

where $k^{*}=\lfloor p / 2\rfloor$,

$$
r_{j}= \begin{cases}1, & j=1, \\ \left\lfloor\frac{p-j+2}{2}\right\rfloor, & j=2, \ldots, p .\end{cases}
$$

Then, following a similar approach to the one used for $W_{3}$ and $\Lambda_{3}$, in Section 4, we obtain near-exact distributions with a somewhat similar structure to those in that section, now with

$$
\begin{aligned}
r= & \frac{(p+1) \bmod 2}{2}+\sum_{j=1}^{p-k^{*}} \frac{j-1}{p} \\
& +\sum_{j=p-k^{*}+1}^{p}\left(\frac{j-1}{p}-\frac{1}{2}\right)=\frac{p-p \bmod 2}{4}
\end{aligned}
$$

and with $\lambda^{*}$ determined as the solution of a system of equations similar to the one in (40), with $\Phi_{W_{3}, 1}$ replaced by $\Phi_{W_{5}, 1}$.

This will yield for $W_{5}$ near-exact distributions which are mixtures of $m+1$ GIG or GNIG distributions, according to the fact that $r$ is an integer or a noninteger, of depth $p+\lfloor(p-1) / 2\rfloor$, with shape parameters

$$
\underbrace{1, \ldots, 1}_{\lfloor(p-1) / 2\rfloor-1}, r_{1}, \ldots, r_{p}, r+j \quad(j=0, \ldots, m),
$$

with $r_{j}$ by (82) and $r$ given by (83), and corresponding rate parameters

$$
\begin{aligned}
& \underbrace{\frac{n-1+2 / p}{2}, \ldots, \frac{n-1+2\lfloor(p-1) / 2\rfloor-2}{2}}_{\lfloor(p-1) / 2\rfloor-1, \text { with step } 2 / p}, \\
& \underbrace{\frac{n-1}{2}, \ldots, \frac{n-p}{2}}_{p}, \lambda^{*},
\end{aligned}
$$

with p.d.f.s and c.d.f.s, respectively, given by

$$
\begin{aligned}
& f_{W_{5}}^{*}(w)=\sum_{j=0}^{m} \pi_{j} f^{\mathrm{GNIG}}(w \mid \underbrace{1, \ldots, 1}_{\lfloor(p-1) / 2\rfloor-1}, r_{1}, \ldots, r_{p}, r \\
& +j ; \underbrace{\frac{n-1+2 / p}{2}, \ldots, \frac{n-1+2\lfloor(p-1) / 2\rfloor-2}{2}}_{\lfloor(p-1) / 2\rfloor-1, \text { with step } 2 / p}, \\
& \underbrace{\frac{n-1}{2}, \ldots, \frac{n-p}{2}}_{p}, \lambda^{*} ; p+\left\lfloor\frac{p-1}{2}\right\rfloor) \text {, } \\
& F_{W_{5}}^{*}(w)=\sum_{j=0}^{m} \pi_{j} F^{\mathrm{GNIG}}(w \mid \underbrace{1, \ldots, 1}_{\lfloor(p-1) / 2\rfloor-1}, r_{1}, \ldots, r_{p}, r \\
& +j ; \underbrace{\frac{n-1+2 / p}{2}, \ldots, \frac{n-1+2\lfloor(p-1) / 2\rfloor-2}{2}}_{\lfloor(p-1) / 2\rfloor-1, \text { with step } 2 / p}, \\
& \underbrace{\frac{n-1}{2}, \ldots, \frac{n-p}{2}}_{p}, \lambda^{*} ; p+\left\lfloor\frac{p-1}{2}\right\rfloor) \text {, }
\end{aligned}
$$

and for $\Lambda_{5}$ with p.d.f.s and c.d.f.s, respectively, given by

$$
\begin{aligned}
& f_{\Lambda_{5}}^{*}(w)=\sum_{j=0}^{m} \pi_{j} f^{\mathrm{GNIG}}(-\log \ell \mid \underbrace{1, \ldots, 1}_{\lfloor(p-1) / 2\rfloor-1}, r_{1}, \ldots, r_{p}, r \\
& +j ; \underbrace{\frac{n-1+2 / p}{2}, \ldots, \frac{n-1+2\lfloor(p-1) / 2\rfloor-2}{2}}_{\lfloor(p-1) / 2\rfloor-1, \text { with step } 2 / p}, \\
& \underbrace{\frac{n-1}{2}, \ldots, \frac{n-p}{2}}_{p}, \lambda^{*} ; p+\left\lfloor\frac{p-1}{2}\right\rfloor) \frac{1}{\ell} \text {, } \\
& F_{\Lambda_{5}}^{*}(w)=\sum_{j=0}^{m} \pi_{j}\left(1-F^{\mathrm{GNIG}}(-\log \ell \mid \underbrace{1, \ldots, 1}_{\lfloor(p-1) / 2\rfloor-1}, r_{1},\right.
\end{aligned}
$$


TABLE 8: Values of the measure $\Delta$ in (5), for the near-exact distributions of the l.r. test statistic $\Lambda_{5}$ in (76), which match $m$ exact moments, for different values of $p$ and samples of size $n=p+1,50,450$.

\begin{tabular}{|c|c|c|c|c|c|c|}
\hline \multirow{2}{*}{$p$} & \multirow{2}{*}{$n$} & \multicolumn{5}{|c|}{$m$} \\
\hline & & 2 & 4 & 6 & 10 & 20 \\
\hline \multirow[t]{3}{*}{10} & 11 & $1.64 \times 10^{-8}$ & $1.39 \times 10^{-12}$ & $4.04 \times 10^{-16}$ & $3.00 \times 10^{-23}$ & $9.74 \times 10^{-33}$ \\
\hline & 60 & $7.64 \times 10^{-10}$ & $6.34 \times 10^{-15}$ & $1.40 \times 10^{-19}$ & $2.69 \times 10^{-28}$ & $1.99 \times 10^{-45}$ \\
\hline & 460 & $1.92 \times 10^{-12}$ & $2.93 \times 10^{-19}$ & $1.15 \times 10^{-25}$ & $6.96 \times 10^{-38}$ & $2.56 \times 10^{-63}$ \\
\hline \multirow[t]{3}{*}{15} & 16 & $2.28 \times 10^{-10}$ & $5.87 \times 10^{-15}$ & $1.29 \times 10^{-18}$ & $4.62 \times 10^{-24}$ & $1.31 \times 10^{-35}$ \\
\hline & 65 & $4.07 \times 10^{-11}$ & $4.01 \times 10^{-16}$ & $1.45 \times 10^{-20}$ & $1.66 \times 10^{-28}$ & $3.79 \times 10^{-43}$ \\
\hline & 465 & $1.38 \times 10^{-13}$ & $3.10 \times 10^{-20}$ & $2.73 \times 10^{-26}$ & $3.09 \times 10^{-37}$ & $6.60 \times 10^{-61}$ \\
\hline \multirow[t]{3}{*}{25} & 26 & $1.35 \times 10^{-11}$ & $5.36 \times 10^{-17}$ & $5.76 \times 10^{-22}$ & $1.44 \times 10^{-29}$ & $2.91 \times 10^{-45}$ \\
\hline & 75 & $7.41 \times 10^{-12}$ & $1.74 \times 10^{-17}$ & $1.62 \times 10^{-22}$ & $1.83 \times 10^{-31}$ & $5.01 \times 10^{-49}$ \\
\hline & 475 & $4.09 \times 10^{-14}$ & $2.98 \times 10^{-21}$ & $9.09 \times 10^{-28}$ & $1.49 \times 10^{-39}$ & $3.64 \times 10^{-65}$ \\
\hline \multirow[t]{3}{*}{50} & 51 & $1.73 \times 10^{-12}$ & $2.51 \times 10^{-19}$ & $1.28 \times 10^{-25}$ & $7.57 \times 10^{-38}$ & $8.22 \times 10^{-64}$ \\
\hline & 100 & $3.12 \times 10^{-12}$ & $5.88 \times 10^{-19}$ & $3.52 \times 10^{-25}$ & $2.54 \times 10^{-37}$ & $8.43 \times 10^{-65}$ \\
\hline & 500 & $4.28 \times 10^{-14}$ & $4.66 \times 10^{-22}$ & $1.59 \times 10^{-29}$ & $3.78 \times 10^{-44}$ & $1.17 \times 10^{-76}$ \\
\hline
\end{tabular}

$$
\begin{aligned}
& \ldots, r_{p}, r+j ; \\
& \underbrace{\frac{n-1+2 / p}{2}, \ldots, \frac{n-1+2\lfloor(p-1) / 2\rfloor-2}{2}}, \\
& \underbrace{\frac{n-1}{2}, \ldots, \frac{n-p}{2}}_{((p-1) / 2\rfloor-1, \text { with step } 2 / p}, \lambda^{*} ; p+\left\lfloor\frac{p-1}{2}\right\rfloor)) .
\end{aligned}
$$

We should note that as it happens with $\Lambda_{3}$ and $W_{3}$, also for $W_{5}$ and $\Lambda_{5}, r$ may be either an integer or a half-integer, so that, in those cases where $r$ is an integer, the near-exact distributions are mixtures of GIG distributions, while when $r$ is noninteger, they are mixtures of GNIG distributions.

In Table 8 are displayed the values of the measure $\Delta$ in (5) for the near-exact distributions developed for $W_{5}$ and $\Lambda_{5}$ and in Table 9 we may find the chi-square asymptotic quantiles for $n W_{5}$, based on a chi-square distribution with $p(p+3) / 2-$ 2 degrees of freedom and the quantiles for the near-exact distributions with $m=2,6$, and 10. Similar conclusions to those drawn for the asymptotic and near-exact distributions for the l.r. statistics in the previous sections also apply here.

\section{The Likelihood Ratio Test for the Simultaneous Test of Nullity of Means and Sphericity of the Covariance Matrix}

We now assume $\underline{X} \sim N_{p}(\underline{\mu}, \Sigma)$, and we now want to test the null hypothesis

$$
\begin{aligned}
H_{0}: \underline{\mu} & =\underline{0} \\
\Sigma & =\sigma^{2} I_{p} \quad\left(\text { with } \sigma^{2} \text { unspecified }\right),
\end{aligned}
$$

which may be written as

$$
H_{0} \equiv H_{02 \mid 01} \circ H_{01}
$$

where

$$
\begin{aligned}
H_{02 \mid 01}: \underline{\mu} & =\underline{0}, \text { assuming } \Sigma=\sigma^{2} I_{p}, \\
H_{01} & : \Sigma=\sigma^{2} I_{p} .
\end{aligned}
$$

For a sample of size $n$, the $(2 / n)$ th power of the l.r. statistic to test $H_{02 \mid 01}$, versus an alternative hypothesis that assumes sphericity for the covariance matrix and no structure for the mean vector, may be shown to be (see Appendix D for details)

$$
\Lambda_{2 \mid 1}=\left(\frac{\operatorname{tr}(A)}{\operatorname{tr}\left(A_{0}\right)}\right)^{p},
$$

where $A$ is the matrix in (27) and now

$$
A_{0}=X^{\prime} X
$$

which was already used in Section 5.

We now have

$$
\begin{aligned}
\operatorname{tr}\left(A_{0}\right) & =\sum_{j=1}^{p} \sum_{i=1}^{n}\left(X_{i j}\right)^{2} \\
& =\underbrace{\sum_{j=1}^{p} \sum_{i=1}^{n}\left(X_{i j}-\bar{X}_{j}\right)^{2}}_{A^{*}}+\underbrace{n \sum_{j=1}^{p}\left(\bar{X}_{j}\right)^{2}}_{B^{* *}},
\end{aligned}
$$

where $A^{*}$ is the r.v. defined in (65), $X=\left[X_{i j}\right](i=$ $1, \ldots, n ; j=1, \ldots, p)$ is the sample matrix, and

$$
\bar{X}_{j}=\frac{1}{n} \sum_{i=1}^{n} X_{i j}
$$




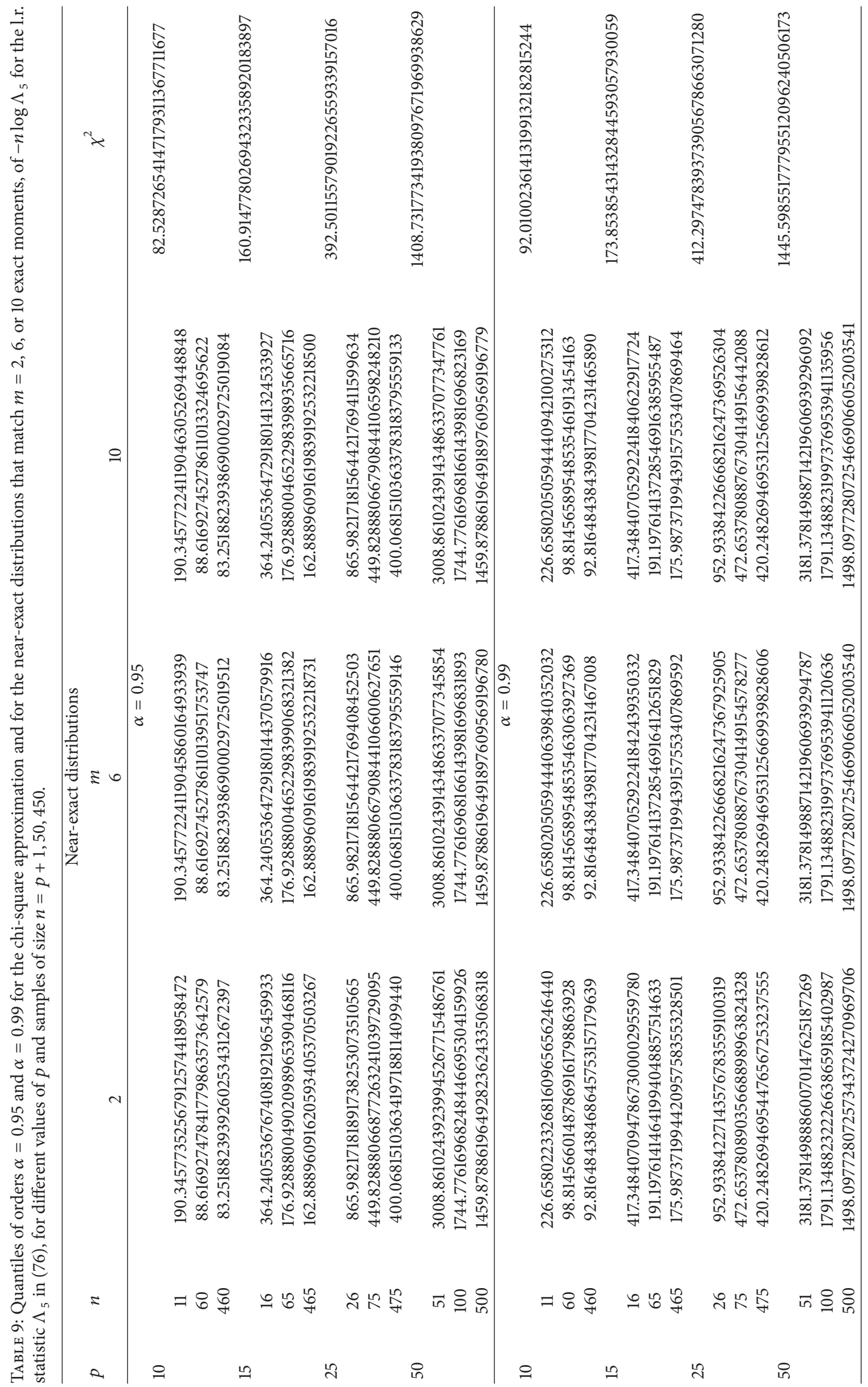


In (93), from standard theory on normal r.v.s, since under $H_{02 \mid 01}$ we have $\bar{X}_{j} \sim N\left(0, \sigma^{2} / n\right)$, independent for $j=1, \ldots, p$, under this null hypothesis,

$$
\frac{\sum_{j=1}^{p}\left(\bar{X}_{j}\right)^{2}}{\sigma^{2} / n}=\frac{B^{* *}}{\sigma^{2}} \sim \chi_{p}^{2} .
$$

Since $A^{*}$ and $B^{* *}$ are independent, given that $A^{*}$ is independent of all $\bar{X}_{j}(j=1, \ldots, p)$ and $B^{* *}$ is defined only from $\bar{X}_{j}$, then, given the distribution of $A^{*}$ in (69),

$$
\begin{aligned}
\frac{\operatorname{tr}(A)}{\operatorname{tr}\left(A_{0}\right)} & =\frac{A^{*}}{A^{*}+B^{* *}} \sim \operatorname{Beta}\left(\frac{(n-1) p}{2}, \frac{p}{2}\right), \\
\Lambda_{2 \mid 1} & \sim\left(\operatorname{Beta}\left(\frac{(n-1) p}{2}, \frac{p}{2}\right)\right)^{p} .
\end{aligned}
$$

Since $H_{01}$ is the same hypothesis as $H_{01}$ in Section 6, the $(2 / n)$ th power of the l.r. statistic to test $H_{0}$ in (90) is thus

$$
\Lambda_{6}=\Lambda_{2 \mid 1} \Lambda_{1}=\frac{|A|}{\left(\operatorname{tr}\left((1 / p) A_{0}\right)\right)^{p}},
$$

where $A$ is given by (27) and $A_{0}$ by (92) and where, from Theorem 5 in [10], we may assure the independence between $\Lambda_{2 \mid 1}$ and $\Lambda_{1}$, so that

$$
\Lambda_{6} \stackrel{d}{\equiv}\left\{\prod_{j=2}^{p} Y_{j}\right\} Y^{*},
$$

where all r.v's are independent, $Y_{j}$ are the r.v's in (75), and $Y^{*}$ is a r.v. with the same distribution as $\Lambda_{2 \mid 1}$ in (97).

Then, if we take $W_{6}=-\log \Lambda_{6}, W_{2}=-\log \Lambda_{2 \mid 1}$, and $W_{1}=-\log \Lambda_{1}$, we may write

$$
\Phi_{W_{6}}(t)=\Phi_{W_{1}}(t) \Phi_{W_{2}}(t),
$$

where, using (36), we may write, for even $p$,

$$
\begin{aligned}
& \Phi_{W_{2}}(t)=E\left(e^{t W_{2}}\right)=E\left(e^{-t \log \Lambda_{2 \mid 1}}\right)=E\left(\Lambda_{2 \mid 1}^{-i t}\right) \\
& =\frac{\Gamma(n p / 2) \Gamma((n p-p) / 2-p i t)}{\Gamma(n p / 2-p i t) \Gamma((n p-p) / 2)} \\
& =\prod_{\ell=0}^{p / 2-1}\left(\frac{n p-p}{2}+\ell\right)\left(\frac{n p-p}{2}-p i t+\ell\right)^{-1} \\
& =\prod_{j=0}^{p / 2-1}\left(\frac{n p-p+2 j}{2}\right)\left(\frac{n p-p+2 j}{2}-p i t\right)^{-1} \\
& =\prod_{j=0}^{p / 2-1}\left(\frac{n-1+(2 j) / p}{2}\right)^{-1} \\
& \quad \cdot\left(\frac{n-1+(2 j) / p}{2}-i t\right)^{-1}
\end{aligned}
$$

while, for odd $p$, we may write

$$
\begin{aligned}
\Phi_{W_{2}}(t) & =\frac{\Gamma(n p / 2) \Gamma((n p-p) / 2-p i t)}{\Gamma(n p / 2-p i t) \Gamma((n p-p) / 2)}=\frac{\Gamma(n p / 2) \Gamma((n p-1) / 2-p i t) \Gamma((n p-1) / 2) \Gamma((n p-p) / 2-p i t)}{\Gamma(n p / 2-p i t) \Gamma((n p-1) / 2)} \frac{\Gamma((n p-1) / 2-p i t) \Gamma((n p-p) / 2)}{\Gamma(n)} \\
& =\frac{\Gamma(n p / 2) \Gamma((n p-1) / 2-p i t)}{\Gamma(n p / 2-p i t) \Gamma((n p-1) / 2)}\left\{\prod_{\ell=0}^{(p-1) / 2-1}\left(\frac{n p-p}{2}+\ell\right)\left(\frac{n p-p}{2}-p i t+\ell\right)^{-1}\right\} \\
& =\frac{\Gamma(n p / 2) \Gamma((n p-1) / 2-p i t)}{\Gamma(n p / 2-p i t) \Gamma((n p-1) / 2)}\left\{\prod_{j=0}^{(p-1) / 2-1}\left(\frac{n-1+(2 j) / p}{2}\right)\left(\frac{n-1+(2 j) / p}{2}-i t\right)^{-1}\right\} .
\end{aligned}
$$

Following then a similar procedure to the one used in the previous section, in order to build near-exact distributions for
$W_{6}$ and $\Lambda_{6}$, we take for $\Phi_{W_{1}}(t)$ the expression for $\Phi_{W_{3}}(t)$ in (A.6) in [6] and write

$$
\begin{aligned}
& \Phi_{W_{6}}(t) \\
& =\underbrace{\left\{\prod_{j=1}^{p-k^{*}} \frac{\Gamma((n-1) / 2+(j-1) / p) \Gamma((n-1) / 2-i t)}{\Gamma((n-1) / 2+(j-1) / p-i t) \Gamma((n-1) / 2)}\right\}\left\{\prod_{j=p-k^{*}+1}^{p} \frac{\Gamma((n-1) / 2+(j-1) / p) \Gamma(n / 2-i t)}{\Gamma((n-1) / 2+(j-1) / p-i t) \Gamma(n / 2)}\right\}\left(\frac{\Gamma(n p / 2) \Gamma((n p-1) / 2-p i t)}{\Gamma(n p / 2-p i t) \Gamma((n p-1) / 2)}\right)^{p \bmod 2}}_{\Phi_{W_{6}, 1}(t)} \\
& \underbrace{\left\{\prod_{j=1}^{p}\left(\frac{n-j}{2}\right)^{r_{j}}\left(\frac{n-j}{2}-i t\right)^{-r_{j}}\right\}\left\{\prod_{j=1}^{\lfloor p / 2\rfloor-1}\left(\frac{n-1+(2 j) / p}{2}\right)\left(\frac{n-1+(2 j) / p}{2}-i t\right)^{-1}\right\}}_{\Phi_{W_{6}, 2}(t)},
\end{aligned}
$$

where $k^{*}=\lfloor p / 2\rfloor$, and the $r_{j}$ are defined in (82). 
Then, we may obtain near-exact distributions for $W_{6}$ and $\Lambda_{6}$, with a similar structure to those in the previous section, now with

$$
\begin{aligned}
r & =\frac{p \bmod 2}{2}+\sum_{j=1}^{p-k^{*}} \frac{j-1}{p}+\sum_{j=p-k^{*}+1}^{p}\left(\frac{j-1}{p}-\frac{1}{2}\right) \\
& =\frac{p-2+3(p \bmod 2)}{4},
\end{aligned}
$$

with $\lambda^{*}$ determined as the solution of a system of equations similar to the one in (40), with $\Phi_{W_{3}, 1}$ replaced by $\Phi_{W_{6}, 1}$.

This yields for $W_{6}$ near-exact distributions which are mixtures of $m+1$ GIG or GNIG distributions, according to the fact that $r$ is an integer or a noninteger, of depth $p+\lfloor p / 2\rfloor$, with shape parameters

$$
\underbrace{1, \ldots, 1}_{\lfloor p / 2\rfloor-1}, r_{1}, \ldots, r_{p}, r+j \quad(j=0, \ldots, m)
$$

with $r_{j}$ by (82) and $r$ given by (104), and corresponding rate parameters

$$
\begin{aligned}
& \underbrace{\frac{n-1+2 / p}{2}, \ldots, \frac{n-1+2\lfloor p / 2\rfloor-2}{2}}_{\lfloor p / 2\rfloor-1, \text { with step } 2 / p}, \\
& \underbrace{\frac{n-1}{2}, \ldots, \frac{n-p}{2}}_{p}, \lambda^{*},
\end{aligned}
$$

with p.d.f.'s and c.d.f.s, respectively, given by

$$
\begin{aligned}
& f_{W_{6}}^{*}(w)=\sum_{j=0}^{m} \pi_{j} f^{\mathrm{GNIG}}(w \mid \underbrace{1, \ldots, 1}_{\lfloor p / 2\rfloor-1}, r_{1}, \ldots, r_{p}, r \\
& +j ; \underbrace{\frac{n-1+2 / p}{2}, \ldots, \frac{n-1+2\lfloor p / 2\rfloor-2}{2}}_{\lfloor p / 2\rfloor-1, \text { with step } 2 / p}, \\
& \underbrace{\frac{n-1}{2}, \ldots, \frac{n-p}{2}}_{p}, \lambda^{*} ; p+\left\lfloor\frac{p}{2}\right\rfloor) \text {, } \\
& F_{W_{6}}^{*}(w)=\sum_{j=0}^{m} \pi_{j} F^{\mathrm{GNIG}}(w \mid \underbrace{1, \ldots, 1}_{\lfloor p / 2\rfloor-1}, r_{1}, \ldots, r_{p}, r \\
& +j ; \underbrace{\frac{n-1+2 / p}{2}, \ldots, \frac{n-1+2\lfloor p / 2\rfloor-2}{2}}_{\lfloor p / 2\rfloor-1, \text { with step } 2 / p}, \\
& \underbrace{\frac{n-1}{2}, \ldots, \frac{n-p}{2}}_{p}, \lambda^{*} ; p+\left\lfloor\frac{p}{2}\right\rfloor) \text {, }
\end{aligned}
$$

and for $\Lambda_{6}$ with p.d.f.s and c.d.f.s, respectively, given by

$$
\begin{gathered}
f_{\Lambda_{6}}^{*}(w)=\sum_{j=0}^{m} \pi_{j} f^{\mathrm{GNIG}}(-\log \ell \mid \underbrace{1, \ldots, 1}_{\lfloor p / 2\rfloor-1}, r_{1}, \ldots, r_{p}, r \\
+j ; \underbrace{\frac{n-1+2 / p}{2}, \ldots, \frac{n-1+2\lfloor p / 2\rfloor-2}{2},}_{p} \\
\underbrace{\left.\frac{n-1}{2}, \ldots, \frac{n-p}{2}, \lambda^{*} ; p+\left\lfloor\frac{p}{2}\right\rfloor\right) \frac{1}{\ell},}_{F_{\Lambda_{6}}} \\
\quad \ldots \sum_{j=0}^{m} \pi_{j}\left(1-F^{\mathrm{GNIG}}(-\log \ell \mid \underbrace{1, \ldots, 1}_{\lfloor p / 2\rfloor-1}, r_{1},\right. \\
\underbrace{\left.\frac{n-1}{2}, \ldots, \frac{n-p}{2}, \lambda^{*} ; p+\left\lfloor\frac{p}{2}\right\rfloor\right)}_{p}) .
\end{gathered}
$$

We should note that as it happens with the statistics in Sections 3-6, also for $W_{6}$ and $\Lambda_{6}, r$ may be either an integer or a half-integer, so that, in those cases where $r$ is an integer, the near-exact distributions are mixtures of GIG distributions, while when $r$ is noninteger, they are mixtures of GNIG distributions.

In Tables 10 and 11 are displayed the values of the measure $\Delta$ in (5) for the near-exact distributions developed for $W_{6}$ and $\Lambda_{6}$ and the chi-square asymptotic quantiles for $n W_{6}$, based on a chi-square distribution with $p(p+3) / 2-1$ degrees of freedom, together with the quantiles for the near-exact distributions with $m=2,6$, and 10. In these tables we may observe the same developments discovered in previous sections. Although in Table 10 we may observe a slight increase in the values of the measure $\Delta$ when we go from $p=10$ to $p=15$ as well as when we compare the near-exact distributions that match only 2 exact moments for $p=10$ and $p=25$, the near-exact distributions developed end up having a sharp asymptotic behavior for increasing $p$, which is clearly visible when we compare the values of $\Delta$ for $p=50$ with those for any other $p$.

\section{Conclusions}

The near-exact approximations developed in this paper allow the practical and precise implementation of simultaneous tests on conditions on mean vectors and of patterns on covariance matrices. These approximations are based on mixtures of Generalized Near-Integer Gamma or Generalized Integer Gamma distributions which are highly manageable and for which there are computational modules available on the Internet: https://sites.google.com/site/nearexactdistributions/ home. Numerical studies show the quality and accuracy of 
TABLE 10: Values of the measure $\Delta$ in (5), for the near-exact distributions of the 1.r. test statistic $\Lambda_{6}$ in (98), which match $m$ exact moments, for different values of $p$ and samples of size $n=p+1,50,450$.

\begin{tabular}{ccccccc}
\hline$p$ & $n$ & 2 & 4 & $m$ & 6 & 10 \\
\hline 10 & 11 & $1.14 \times 10^{-10}$ & $5.65 \times 10^{-14}$ & $6.84 \times 10^{-17}$ & $3.17 \times 10^{-22}$ & $3.95 \times 10^{-33}$ \\
& 60 & $2.53 \times 10^{-11}$ & $2.02 \times 10^{-16}$ & $4.05 \times 10^{-21}$ & $7.58 \times 10^{-30}$ & $5.45 \times 10^{-45}$ \\
& 460 & $6.89 \times 10^{-14}$ & $1.10 \times 10^{-20}$ & $5.17 \times 10^{-27}$ & $1.29 \times 10^{-38}$ & $3.73 \times 10^{-64}$ \\
\hline 15 & 16 & $2.69 \times 10^{-9}$ & $5.09 \times 10^{-14}$ & $5.26 \times 10^{-18}$ & $3.34 \times 10^{-24}$ & $3.53 \times 10^{-36}$ \\
& 65 & $4.00 \times 10^{-10}$ & $1.68 \times 10^{-15}$ & $4.58 \times 10^{-20}$ & $3.83 \times 10^{-28}$ & $2.62 \times 10^{-43}$ \\
& 465 & $1.33 \times 10^{-12}$ & $1.25 \times 10^{-19}$ & $7.40 \times 10^{-26}$ & $3.82 \times 10^{-37}$ & $9.27 \times 10^{-61}$ \\
\hline 25 & 26 & $1.30 \times 10^{-10}$ & $3.24 \times 10^{-16}$ & $4.27 \times 10^{-21}$ & $7.05 \times 10^{-30}$ & $2.14 \times 10^{-45}$ \\
& 75 & $6.66 \times 10^{-11}$ & $9.02 \times 10^{-17}$ & $6.11 \times 10^{-22}$ & $2.92 \times 10^{-31}$ & $3.64 \times 10^{-49}$ \\
& 475 & $3.61 \times 10^{-13}$ & $1.52 \times 10^{-20}$ & $3.12 \times 10^{-27}$ & $1.76 \times 10^{-39}$ & $4.59 \times 10^{-65}$ \\
\hline 50 & 51 & $1.78 \times 10^{-13}$ & $2.02 \times 10^{-20}$ & $5.12 \times 10^{-27}$ & $1.83 \times 10^{-39}$ & $1.14 \times 10^{-63}$ \\
& 100 & $3.32 \times 10^{-13}$ & $4.87 \times 10^{-20}$ & $1.59 \times 10^{-26}$ & $1.82 \times 10^{-38}$ & $7.39 \times 10^{-64}$ \\
& 500 & $4.68 \times 10^{-15}$ & $3.93 \times 10^{-23}$ & $7.64 \times 10^{-31}$ & $3.73 \times 10^{-45}$ & $6.76 \times 10^{-77}$ \\
\hline
\end{tabular}

near-exact distributions developed, contrary to what happens, for example, with usual chi-square approximation. A natural extension of this work will be to develop approximations that will allow the implementation of simultaneous tests on the equality or nullity of several mean vectors and on the equality of the corresponding covariance matrices to a given matrix which may have a specific structure.

\section{Appendices}

\section{A. On the Likelihood Ratio Test Statistic to Test the Nullity of Means Assuming Compound Symmetry of the Covariance Matrix}

The 1.r. statistic to test the null hypotheses

$$
\begin{aligned}
H_{0}: \underline{\mu} & =\underline{0}, \\
\Sigma & =\Sigma_{c s}
\end{aligned}
$$

versus $H_{1}: \Sigma=\Sigma_{c s}($ and any $\underline{\mu})$

is the l.r. statistic to test the null hypothesis $H_{02 \mid 01}$ in (46), which is

$$
\Lambda_{2 \mid 1}^{*}=\frac{\max L_{0}}{\max L_{1}}
$$

where $L_{0}$, the likelihood function under $H_{0}$ in (A.1) above, is, for $\Sigma_{c s}$, defined as in (3), for which we have $\left|\Sigma_{c s}\right|=(a-$ b) $)^{p-1}(a+(p-1) b)$,

$$
\begin{aligned}
L_{0}= & (2 \pi)^{-n p / 2}\left((a-b)^{p-1}(a+(p-1) b)\right)^{-n / 2} \\
& \cdot e^{-(1 / 2) \operatorname{tr}\left[X^{\prime} X \Sigma_{c s}^{-1}\right]},
\end{aligned}
$$

where $X$ is the $n \times p$ sample matrix, and $L_{1}$, the likelihood function under $H_{1}$ in (A.1), is

$$
\begin{aligned}
L_{1}= & (2 \pi)^{-n p / 2}\left((a-b)^{p-1}(a+(p-1) b)\right)^{-n / 2} \\
& \cdot e^{-(1 / 2) \operatorname{tr}\left[\left(X-E_{n 1} \underline{\mu}^{\prime}\right)^{\prime}\left(X-E_{n 1} \underline{\mu}^{\prime}\right) \Sigma_{c s}^{-1}\right]}
\end{aligned}
$$

where $E_{n 1}$ is an $n \times 1$ vector of 1's.

Since under $H_{1}$ we have $\widehat{a}$ and $\widehat{b}$, the m.l.e.'s of $a$ and $b$ given by (28), and the m.l.e. of $\Sigma_{c s}$ is $\widehat{\Sigma}_{c s}=(1 / n)\left(\widehat{a} I_{p}+\widehat{b}\left(E_{p p}-\right.\right.$ $\left.I_{p}\right)$ ), then

$$
\begin{aligned}
& \widehat{\Sigma}_{c s}^{-1}=\frac{n}{\widehat{a}^{2}+(p-2) \hat{a} \widehat{b}-(p-1) \hat{b}^{2}}((\widehat{a}+(p-2) \widehat{b}) \\
& \left.\cdot I_{p}-\widehat{b}\left(E_{p p}-I_{p}\right)\right), \\
& \max L_{1}=(2 \pi)^{-n p / 2}\left((\widehat{a}-\widehat{b})^{p-1}(\widehat{a}+(p-1) \widehat{b})\right)^{-n / 2} \\
& \cdot e^{-(1 / 2) \operatorname{tr}\left[A \widehat{\Sigma}_{c s}^{-1}\right]},
\end{aligned}
$$

where $A=\left(X-E_{n 1} \underline{\bar{X}}^{\prime}\right)\left(X-E_{n 1} \underline{\bar{X}}^{\prime}\right)$ is the matrix defined in (27), with $\underline{\bar{X}}$, the vector sample means, which is the m.l.e. of $\underline{\mu}$.

In (A.6),

$$
\operatorname{tr}\left(A \widehat{\Sigma}_{c s}^{-1}\right)=n
$$

$$
\begin{aligned}
& \frac{1}{\widehat{a}^{2}+(p-2) \hat{a} \widehat{b}-(p-1) \widehat{b}^{2}}\left\{\sum_{j=1}^{p} a_{j j}(\widehat{a}\right. \\
& \left.+(p-2) \hat{b})-\widehat{b} \sum_{\substack{k=1 \\
k \neq j}}^{p} a_{j k}\right\}=n
\end{aligned}
$$




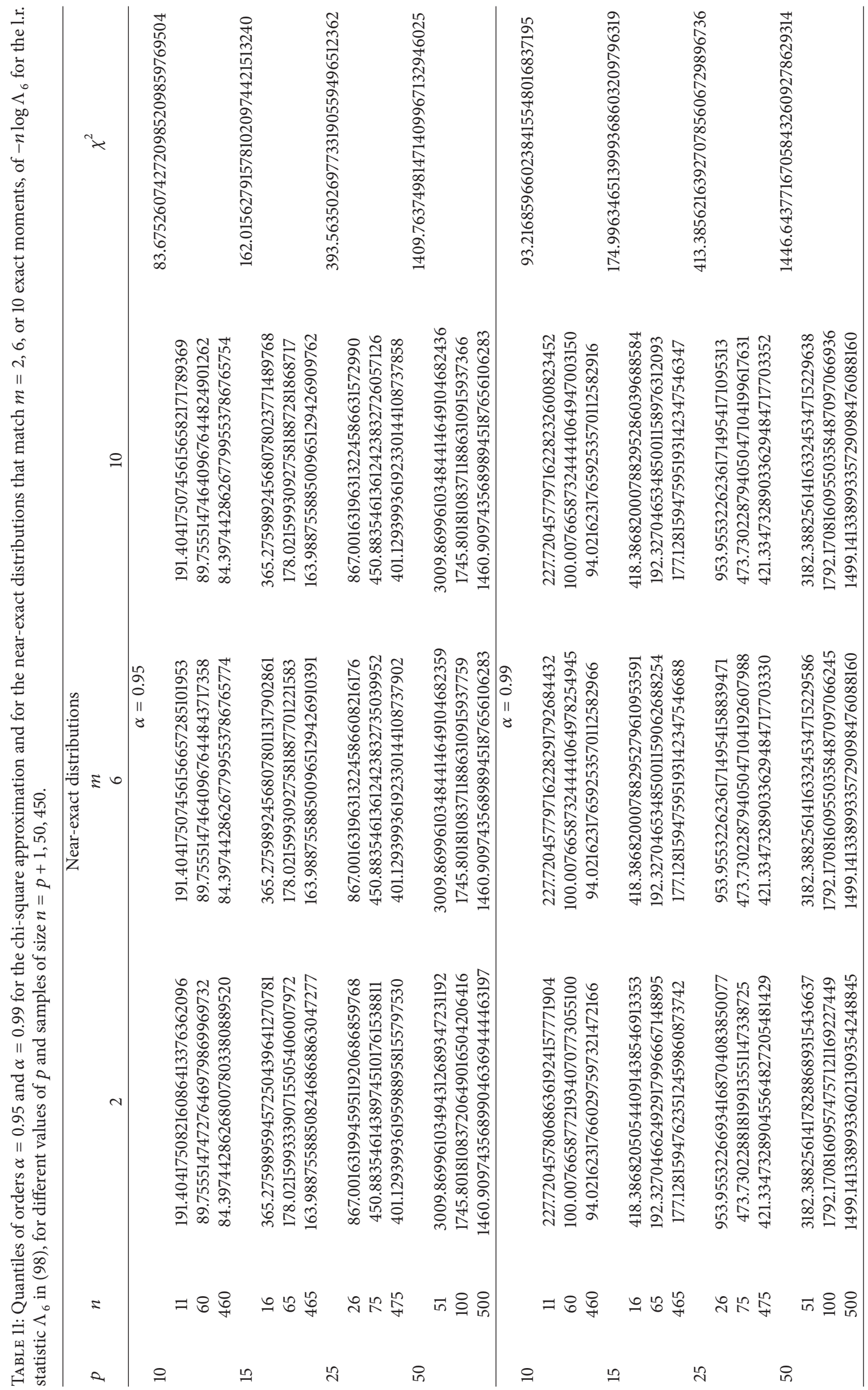




$$
\begin{aligned}
& \cdot \frac{1}{\widehat{a}^{2}+(p-2) \hat{a} \widehat{b}-(p-1) \hat{b}^{2}}\{p \widehat{a}(\widehat{a}+(p-2) \\
& \cdot \widehat{b})-\widehat{b} p(p-1) \hat{b}\}=n p,
\end{aligned}
$$

so that

$$
\begin{aligned}
& \max L_{1} \\
& =(2 \pi)^{-n p / 2}\left((\widehat{a}-\widehat{b})^{p-1}(\widehat{a}+(p-1) \widehat{b})\right)^{-n / 2} e^{-n p / 2} .
\end{aligned}
$$

Under $H_{0}$ the m.l.e.'s of $a$ and $b$ are $\widehat{a}_{0}$ and $\widehat{b}_{0}$, given by (48), and the m.l.e. of $\Sigma_{c s}$ is $\widehat{\Sigma}_{c s(0)}=(1 / n)\left(\widehat{a}_{0} I_{p}+\widehat{b}_{0}\left(E_{p p}-I_{p}\right)\right)$, so that

$$
\begin{aligned}
& \widehat{\Sigma}_{c s(0)}^{-1}=n \frac{1}{\widehat{a}_{0}^{2}+(p-2) \widehat{a}_{0} \widehat{b}_{0}-(p-1) \hat{b}_{0}^{2}}\left(\left(\widehat{a}_{0}\right.\right. \\
& \left.\left.\quad+(p-2) \widehat{b}_{0}\right) I_{p}-\widehat{b}_{0}\left(E_{p p}-I_{p}\right)\right) \\
& \max L_{0}=(2 \pi)^{-n p / 2}\left(( \widehat { a } _ { 0 } - \widehat { b } _ { 0 } ) ^ { p - 1 } \left(\widehat{a}_{0}\right.\right. \\
& \left.\left.\quad+(p-1) \widehat{b}_{0}\right)\right)^{-n / 2} e^{-(1 / 2) \operatorname{tr}\left[A_{0} \widehat{c}_{c s(0)}^{-1}\right]}
\end{aligned}
$$

where $A_{0}$ is the matrix defined in (49).

Given the definition of $\widehat{a}_{0}$ and $\widehat{b}_{0}$ in (48), following similar steps to the ones used under $H_{1}, \operatorname{tr}\left[A_{0} \widehat{\Sigma}_{c s(0)}\right]=n p$, so that finally

$$
\begin{aligned}
\max L_{0}= & (2 \pi)^{-n p / 2} \\
& \cdot\left(\left(\widehat{a}_{0}-\widehat{b}_{0}\right)^{p-1}\left(\widehat{a}_{0}+(p-1) \widehat{b}_{0}\right)\right)^{-n / 2} \\
& \cdot e^{-n p / 2} .
\end{aligned}
$$

As such, we have

$$
\Lambda_{2 \mid 1}^{*}=\left(\frac{(\widehat{a}-\widehat{b})^{p-1}(\widehat{a}+(p-1) \hat{b})}{\left(\widehat{a}_{0}-\widehat{b}_{0}\right)^{p-1}\left(\widehat{a}_{0}+(p-1) \widehat{b}_{0}\right)}\right)^{n / 2}
$$

and its $(2 / n)$ th power given by $\Lambda_{2 \mid 1}$ in (47).

Let $H_{p}$ be a Helmert matrix of order $p$. Then

$$
\begin{aligned}
H_{p} \Sigma_{c s} H_{p}^{\prime} & =\Delta \\
& =\operatorname{diag}(a+(p-1) b, \underbrace{a-b, \ldots, a-b}_{p-1}),
\end{aligned}
$$

and if we take $A^{*}=H_{p} A H_{p}^{\prime}$, we will have

$$
\begin{aligned}
\widehat{a-b} & =\widehat{a}-\widehat{b}=\frac{1}{p-1} \sum_{j=2}^{p} a_{j j}^{*}, \\
a+\widehat{(p-1)} b & =\widehat{a}+(p-1) \hat{b}=a_{11}^{*},
\end{aligned}
$$

where $a_{j j}^{*}(j=1, \ldots, p)$ are the diagonal elements of $A^{*}$.

Since, for $A$ in (27), we have

$$
A \sim W_{p}\left(n-1, \Sigma_{c s}\right)
$$

then

$$
A^{*}=H_{p} A H_{p}^{\prime} \sim W_{p}(n-1, \Delta)
$$

so that the diagonal elements of $A^{*}$ are independent, with

$$
\frac{a_{j j}^{*}}{\Delta_{j}} \sim \chi_{n-1}^{2}, \quad(j=1, \ldots, p)
$$

where $\Delta_{j}$ is the $j$ th diagonal element of $\Delta$.

As such, $\widehat{a-b}=\widehat{a}-\widehat{b}$ is distributed as $(a-b) /(p-1)$ times a chi-square r.v. with $(n-1)(p-1)$ degrees of freedom, independently distributed from $a+\widehat{(p-1)} b=\widehat{a}+(p-1) \widehat{b}$ which is distributed as $a+(p-1) b$ times a chi-square with $n-1$ degrees of freedom.

Concerning the matrix $A_{0}$ in (49), we know that

$$
A_{0} \sim W_{p}\left(n, \Sigma_{c s}\right)
$$

so that

$$
A_{0}^{*}=H_{p} A_{0} H_{p}^{\prime} \sim W_{p}(n, \Delta),
$$

which shows that the diagonal elements of $A_{0}^{*}, a_{0(j j)}^{*}(j=$ $1, \ldots, p)$, are independently distributed, with

$$
\frac{a_{0(j j)}^{*}}{\Delta_{j}} \sim \chi_{n}^{2} .
$$

But then, the m.l.e.s of $a$ and $b$, under $H_{0}$ in (A.1), are given by (48) or, equivalently,

$$
\begin{aligned}
\widehat{a_{0}-b_{0}} & =\widehat{a}_{0}-\widehat{b}_{0}=\frac{1}{p-1} \sum_{j=2}^{p} a_{0(j j)}^{*}, \\
a_{0}+\widehat{(p-1)} b_{0} & =\widehat{a}_{0}+(p-1) \widehat{b}_{0}=a_{0(11)}^{*}
\end{aligned}
$$

as such, with $\widehat{a_{0}-b_{0}}=\widehat{a}_{0}-\widehat{b}_{0}$ distributed as $(a-b) /(p-$ 1) times a chi-square r.v. with $n(p-1)$ degrees of freedom, independently distributed from $a_{0}+\widehat{(p-1)} b_{0}=\widehat{a}_{0}+(p-1) \widehat{b}_{0}$ which is distributed as $a+(p-1) b$ times a chi-square with $n$ degrees of freedom. 
We can write

$$
\begin{aligned}
A & =X^{\prime}\left(I_{n}-\frac{1}{n} E_{n n}\right) X=X^{\prime} X-\frac{1}{n} X^{\prime} E_{n n} X \\
& =A_{0}-\frac{1}{n} X^{\prime} E_{n n} X,
\end{aligned}
$$

or

$$
A_{0}=A+\frac{1}{n} X^{\prime} E_{n n} X
$$

where, by application of Cochran's Theorem (see, e.g., [12, Thm. 7.4.1]), it is easy to show that, under $H_{0}$ in (A.1), $A$ and $(1 / n) X^{\prime} E_{n n} X$ are independent, since we can write

$$
\begin{aligned}
A & =\sum_{i=1}^{n} \underline{X}_{i}\left(I_{n}-\frac{1}{n} E_{n n}\right) \underline{X}_{i}^{\prime}, \\
\frac{1}{n} X^{\prime} E_{n n} X & =\sum_{i=1}^{n} \frac{1}{n} \underline{X}_{i} E_{n n} \underline{X}_{i}^{\prime},
\end{aligned}
$$

where, under the null hypothesis in (A.1), the $i$ th column of $X^{\prime}$ is

$$
\underline{X}_{i} \sim N_{p}\left(\underline{0}, \Sigma_{c s}\right),
$$

and we have

$$
\begin{aligned}
& \left(I_{n}-\frac{1}{n} E_{n n}\right)\left(\frac{1}{n} E_{n n}\right)=\frac{1}{n} E_{n n}-\frac{1}{n^{2}} E_{n n} E_{n n} \\
& =\frac{1}{n} E_{n n}-\frac{1}{n} E_{n n}=0,
\end{aligned}
$$

with

$$
\begin{aligned}
\operatorname{rank}\left(I_{n}-\frac{1}{n} E_{n n}\right) & =\operatorname{tr}\left(I_{n}-\frac{1}{n} E_{n n}\right)=n-1, \\
\operatorname{rank}\left(\frac{1}{n} E_{n n}\right) & =1,
\end{aligned}
$$

which yields for $A$ the distribution in (A.14) and

$$
A_{1}=\frac{1}{n} X^{\prime} E_{n n} X \sim W_{p}\left(1, \Sigma_{c s}\right)
$$

so that

$$
A_{1}^{*}=H_{p} A_{1} H_{p}^{\prime} \sim W_{p}(1, \Delta),
$$

so that, for $j=1, \ldots, p$, each $j$ th diagonal element of $A_{1}^{*}, a_{1(j j)}^{*}$ has a distribution such that

$$
\frac{a_{1(j j)}^{*}}{\Delta_{j}} \sim \chi_{1}^{2},
$$

with

$$
\widehat{a_{0}-b_{0}}=\widehat{a-b}+\widehat{a_{1}-b_{1}}
$$

where

$$
\widehat{a_{1}-b_{1}}=\frac{1}{p-1} \sum_{j=2}^{p} a_{1(j j)}^{*},
$$

so that $\widehat{a_{1}-b_{1}}$ is independent of $\widehat{a-b}$ and it has a distribution which is that of a chi-square with $p-1$ degrees of freedom, multiplied by $(a-b) /(p-1)$, while we also have

$$
a_{0}+\widehat{(p-1)} b_{0}=a+\widehat{(p-1)} b+a_{1}+\widehat{(p-1)} b_{1},
$$

where $a_{1}+\widehat{(p-1)} b_{1}$ is distributed as a chi-square with 1 degree of freedom, multiplied by $a+(p-1) b$, and distributed independently of $a+\widehat{(p-1)} b$.

As such

$$
\begin{aligned}
\frac{\widehat{a}-\widehat{b}}{\widehat{a}_{0}-\widehat{b}_{0}} & =\frac{\widehat{a-b}}{\widehat{a_{0}-b_{0}}} \\
& \sim \operatorname{Beta}\left(\frac{(n-1)(p-1)}{2}, \frac{p-1}{2}\right),
\end{aligned}
$$

which is independently distributed from

$$
\begin{aligned}
\frac{\widehat{a}+(p-1) \hat{b}}{\widehat{a}_{0}+(p-1) \hat{b}_{0}} & =\frac{a+\widehat{(p-1)} b}{a_{0}+\widehat{(p-1)} b_{0}} \\
& \sim \operatorname{Beta}\left(\frac{n-1}{2}, \frac{1}{2}\right),
\end{aligned}
$$

yielding for $\Lambda_{2 \mid 1}$ in (47) the distribution stated in Section 5, as also stated by Geisser in [3], but where the expression for the l.r. statistic should be corrected to be stated as in (A.11).

\section{B. On the Distribution of $\Lambda_{4}$ in (51)}

In order to show the distribution of $\Lambda_{4}$ in (51) as mentioned in (54) and (55), all we need to do is to show that

$$
\left\{\prod_{j=2}^{p} Y_{j}^{*}\right\}\left(Y_{1}\right)^{p-1} \stackrel{d}{\equiv}\left\{\prod_{j=2}^{p} Y_{j}^{* *}\right\},
$$

for $Y_{j}^{*}$ in (53), $Y_{1}$ in (52), and $Y_{j}^{* *}$ in (55), where $Y_{1}$ is independent of all $Y_{j}^{*}$.

Let us take $\Lambda_{1}^{*}$ as a r.v. whose distribution is the same as that of the product of r.v.s on the left hand side of (B.1) and $\Lambda_{2}^{*}$ as a r.v. whose distribution is the same as that of the product of r.v.s on the right hand side of (B.1).

Then, using the multiplication formula for the Gamma function

$$
\Gamma(n z)=(2 \pi)^{(1-n) / 2} n^{n-z-1 / 2} \prod_{k=0}^{n-1} \Gamma\left(z+\frac{k}{n}\right),
$$


we may write

$$
\begin{aligned}
E[ & \left.\left(\Lambda_{1}^{*}\right)^{h}\right] \\
& =\left\{\prod_{j=2}^{p} \frac{\Gamma((n-1) / 2+(j-2) /(p-1)) \Gamma((n-j) / 2+h)}{\Gamma((n-j) / 2) \Gamma((n-1) / 2+(j-2) /(p-1)+h)}\right\} \\
& \cdot \frac{\Gamma(n(p-1) / 2) \Gamma((n-1)(p-1) / 2+(p-1) h)}{\Gamma((n-1)(p-1) / 2) \Gamma(n(p-1) / 2+(p-1) h)} \\
= & \left\{\prod_{j=2}^{p} \frac{\Gamma((n-1) / 2+(j-2) /(p-1)) \Gamma((n-j) / 2+h)}{\Gamma((n-j) / 2) \Gamma((n-1) / 2+(j-2) /(p-1)+h)}\right\} \\
& \cdot\left\{\prod_{j=0}^{p-2} \frac{\Gamma(n / 2+j /(p-1)) \Gamma((n-1) / 2+j /(p-1)+h)}{\Gamma((n-1) / 2+j /(p-1)) \Gamma(n / 2+j /(p-1)+h)}\right\} \\
= & \prod_{j=2}^{p} \frac{\Gamma(n / 2+(j-2) /(p-1)) \Gamma((n-j) / 2+h)}{\Gamma((n-j) / 2) \Gamma(n / 2+(j-2) /(p-1)+h)} \\
= & E\left[\left(\Lambda_{2}^{*}\right)^{h}\right] .
\end{aligned}
$$

But then, since both $\Lambda_{1}^{*}$ and $\Lambda_{2}^{*}$ have a delimited support, more precisely, between zero and 1 , their distribution is determined by their moments and as such $\Lambda_{1}^{*} \stackrel{d}{\equiv} \Lambda_{2}^{*}$.

\section{Derivation of the 1.r. Statistic to Test Equality of Means, Assuming Sphericity of the Covariance Matrix}

Let us suppose that $\underline{X} \sim N_{p}(\mu, \Sigma)$, where $\mu=\left[\mu_{1}, \ldots, \mu_{p}\right]^{\prime}$, and that we want to test the hypotheses

$$
H_{02 \mid 01}: \mu_{1}=\cdots=\mu_{p} \text {, assuming } \Sigma=\sigma^{2} I_{p}
$$

versus $H_{12 \mid 01}: \Sigma=\sigma^{2} I_{p}$ (and no structure for $\underline{\mu}$ ),

based on a sample of size $n$.

Under $H_{12 \mid 01}$, the m.l.e. of $\underline{\mu}$ is

$$
\underline{\widehat{\mu}}=\underline{\bar{X}}=\frac{1}{n} X^{\prime} E_{n 1}
$$

with

$$
\underline{\bar{X}}=\left[\bar{X}_{1}, \ldots, \bar{X}_{p}\right]
$$

where $X$ is the $n \times p$ sample matrix and $E_{n 1}$ is a matrix of dimensions $n \times 1$ of 1's, that is, a column vector of $n$ 1's. Also, under this same hypothesis, the m.l.e. of $\Sigma$ is

$$
\widehat{\Sigma}=\widehat{\sigma}^{2} I_{p}
$$

where

$$
\widehat{\sigma}^{2}=\frac{1}{p} \sum_{j=1}^{p} \frac{1}{n} \sum_{i=1}^{n}\left(X_{i j}-\bar{X}_{j}\right)^{2}=\frac{1}{n p} \operatorname{tr}(A),
$$

where $X_{i j}$ is the element in the $i$ th row and $j$ th column of $X$ and $A$ is the matrix in (27).
Since the likelihood function is

$$
L=(2 \pi)^{-n p / 2}|\Sigma|^{-n / 2} e^{-(1 / 2) \operatorname{tr}\left[\left(X-E_{n 1} \underline{\mu}^{\prime}\right)^{\prime}\left(X-E_{n 1} \underline{\mu}^{\prime}\right) \Sigma^{-1}\right]},
$$

its maximum under $H_{12 \mid 01}$ in (C.1) is thus

$$
\begin{aligned}
& \max L_{1}=L(\underline{\hat{\mu}}, \widehat{\Sigma})=(2 \pi)^{-n p / 2}\left|\widehat{\sigma}^{2} I_{p}\right|^{-n / 2} \\
& \cdot e^{-(1 / 2) \operatorname{tr}\left[\left(X-E_{n 1} \underline{\bar{X}}^{\prime}\right)^{\prime}\left(X-E_{n 1} \underline{\bar{X}}^{\prime}\right)\left(1 / \widehat{\sigma}^{2}\right) I_{p}\right]}=(2 \pi)^{-n p / 2} \\
& \cdot\left(\widehat{\sigma}^{2}\right)^{-n p / 2} e^{-\left(1 / 2 \widehat{\sigma}^{2}\right) \operatorname{tr}\left[\left(X-E_{n 1} \bar{X}^{\prime}\right)^{\prime}\left(X-E_{n 1} \bar{X}^{\prime}\right)\right]} \\
& =(2 \pi)^{-n p / 2}\left(\widehat{\sigma}^{2}\right)^{-n p / 2} e^{-\left(1 / 2 \widehat{\sigma}^{2}\right) \operatorname{tr}(A)}=(2 \pi)^{-n p / 2} \\
& \cdot\left(\frac{\operatorname{tr}(A)}{n p}\right)^{-n p / 2} e^{-n p / 2} .
\end{aligned}
$$

Under the null hypothesis $H_{02 \mid 01}$ in (C.1), the m.l.e. of $\mu$ is

$$
\begin{aligned}
\underline{\underline{\mu}}_{0} & =\underline{\bar{X}}^{*}=\left(\frac{1}{p} \sum_{j=1}^{p} \bar{X}_{j}\right) E_{p 1}=\frac{1}{n p} E_{p p} X^{\prime} E_{n 1} \\
& =\frac{1}{p} E_{p p} \underline{\bar{X}}
\end{aligned}
$$

where $(1 / p) \sum_{j=1}^{p} \bar{X}_{j}=\bar{X}$ and the m.l.e. of $\sum$ is

$$
\widehat{\Sigma}_{0}=\widehat{\sigma}_{0}^{2} I_{p}
$$

with

$$
\widehat{\sigma}_{0}^{2}=\frac{1}{p} \sum_{j=1}^{p} \frac{1}{n} \sum_{i=1}^{n}\left(X_{i j}-\bar{X}\right)^{2}=\frac{1}{p} \operatorname{tr}\left(A_{0}\right),
$$

where $A_{0}$ is the matrix in (62), so that the maximum of the likelihood function under $H_{02 \mid 01}$ in (C.1) is

$$
\begin{aligned}
& \max L_{0}=L\left(\underline{\underline{\mu}}_{0}, \widehat{\Sigma}_{0}\right)=(2 \pi)^{-n p / 2}\left|\widehat{\sigma}_{0}^{2} I_{p}\right|^{-n / 2} \\
& \cdot e^{-(1 / 2) \operatorname{tr}\left[\left(X-E_{n 1} \widehat{\underline{\mu}}_{-0}^{\prime}\right)^{\prime}\left(X-E_{n 1} \underline{\underline{\mu}}_{0}^{\prime}\right)\left(1 / \widehat{\sigma}_{0}^{2}\right) I_{p}\right]}=(2 \pi)^{-n p / 2} \\
& \cdot\left(\widehat{\sigma}_{0}^{2}\right)^{-n p / 2} e^{-\left(1 / 2 \widehat{\sigma}^{2}\right) \operatorname{tr}\left(A_{0}\right)}=(2 \pi)^{-n p / 2} \\
& \cdot\left(\frac{\operatorname{tr}\left(A_{0}\right)}{n p}\right)^{-n p / 2} e^{-n p / 2} .
\end{aligned}
$$

The 1.r. statistic to test the hypotheses in (C.1) is thus

$$
\begin{aligned}
\Lambda_{2 \mid 1}^{*} & =\frac{\max L_{0}}{\max L_{1}}=\frac{\left(\operatorname{tr}\left(A_{0}\right) / n p\right)^{-n p / 2}}{(\operatorname{tr}(A) / n p)^{-n p / 2}} \\
& =\left(\frac{\operatorname{tr}(A)}{\operatorname{tr}\left(A_{0}\right)}\right)^{n p / 2},
\end{aligned}
$$

so that its $(2 / n)$ th power is the statistic $\Lambda_{2 \mid 1}$ in (72). 


\section{Derivation of the 1.r. Statistic to Test the Nullity of Means, Assuming Sphericity of the Covariance Matrix}

Let us suppose that $\underline{X} \sim N_{p}(\mu, \Sigma)$, where $\mu=\left[\mu_{1}, \ldots, \mu_{p}\right]^{\prime}$, and that we want to test the hypotheses

$$
H_{02 \mid 01}: \underline{\mu}=\underline{0} \text {, assuming } \Sigma=\sigma^{2} I_{p}
$$

versus $H_{12 \mid 01}: \Sigma=\sigma^{2} I_{p}($ and no structure for $\underline{\mu})$,

based on a sample of size $n$.

Under $H_{12 \mid 01}$ in (D.1), the m.l.e.s of $\mu$ and $\Sigma$ are the same as those in Appendix $\mathrm{C}$, since this hypothesis is the same as the alternative hypothesis in (C.1) in Appendix C, and, as such, the function $L_{1}$, the maximum of the likelihood function under $H_{12 \mid 01}$ in (D.1), is the same as $L_{1}$ in (C.7) in Appendix C. is

Under the null hypothesis $H_{02 \mid 01}$ in (D.1), the m.l.e. of $\Sigma$

$$
\widehat{\Sigma}_{0}=\widehat{\sigma}_{0}^{2} I_{p}
$$

with

$$
\widehat{\sigma}_{0}^{2}=\frac{1}{p} \sum_{j=1}^{p} \frac{1}{n} \sum_{i=1}^{n}\left(X_{i j}\right)^{2}=\frac{1}{p} \operatorname{tr}\left(A_{0}\right)
$$

where $A_{0}$ is the matrix in (92), so that the maximum of the likelihood function under $H_{02 \mid 01}$ in (D.1) is given by a similar function to that in (C.11), now with $A_{0}$ given by (92) and $\widehat{\sigma}_{0}^{2}$ given by (D.3).

The l.r. statistic to test the hypotheses in (D.1) is thus, for $A$ in (27) and $A_{0}$ in (92),

$$
\begin{aligned}
\Lambda_{2 \mid 1}^{*} & =\frac{\max L_{0}}{\max L_{1}}=\frac{\left(\operatorname{tr}\left(A_{0}\right) / n p\right)^{-n p / 2}}{(\operatorname{tr}(A) / n p)^{-n p / 2}} \\
& =\left(\frac{\operatorname{tr}(A)}{\operatorname{tr}\left(A_{0}\right)}\right)^{n p / 2},
\end{aligned}
$$

so that its $(2 / n)$ th power is the statistic $\Lambda_{2 \mid 1}$ in (91).

\section{Conflict of Interests}

The authors declare that there is no conflict of interests regarding the publication of this paper.

\section{Acknowledgment}

Research was partially supported by National Funds through FCT Fundação para a Ciência e a Tecnologia, project UID/ MAT/00297/2013 (CMA/UNL).

\section{References}

[1] I. Olkin and S. J. Press, "Testing and estimation for a circular stationary model," Annals of Mathematical Statistics, vol. 40, pp. 1358-1373, 1969.
[2] S. S. Wilks, "Sample criteria for testing equality of means, equality of variances, and equality of covariances in a normal multivariate distribution," Annals of Mathematical Statistics, vol. 17, pp. 257-281, 1946.

[3] S. Geisser, "Multivariate analysis of variance for a special covariance case," Journal of the American Statistical Association, vol. 58, pp. 660-669, 1963.

[4] C. A. Coelho, F. J. Marques, and S. Oliveira, "The exact distribution of the likelihood ratio test statistic used to test simultaneously the equality of means and circularity of the covariance matrix," AIP Conference Proceedings, vol. 1558, pp. 789-792, 2013.

[5] C. A. Coelho, “The generalized near-integer Gamma distribution: a basis for 'near-exact' approximations to the distribution of statistics which are the product of an odd number of independent Beta random variables," Journal of Multivariate Analysis, vol. 89, no. 2, pp. 191-218, 2004.

[6] F. J. Marques, C. A. Coelho, and B. C. Arnold, "A general nearexact distribution theory for the most common likelihood ratio test statistics used in multivariate analysis," Test, vol. 20, no. 1, pp. 180-203, 2011.

[7] C. A. Coelho, "The generalized integer gamma distribution-a basis for distributions in multivariate statistics," Journal of Multivariate Analysis, vol. 64, no. 1, pp. 86-102, 1998.

[8] J. L. Fields, "A note on the asymptotic expansion of a ratio of Gamma functions," Proceedings of the Edinburgh Mathematical Society-Series II, vol. 15, pp. 43-45, 1966.

[9] F. G. Tricomi and A. Erdélyi, "The asymptotic expansion of a ratio of gamma functions," Pacific Journal of Mathematics, vol. 1, no. 1, pp. 133-142, 1951.

[10] S. T. Jensen, "Covariance hypotheses which are linear in both the covariance and the inverse covariance," The Annals of Statistics, vol. 16, no. 1, pp. 302-322, 1988.

[11] J. W. Mauchly, "Significance test for sphericity of a normal $n$ variate distribution," Annals of Mathematical Statistics, vol. 11, pp. 204-209, 1940.

[12] T. W. Anderson, An Introduction to Multivariate Statistical Analysis, John Wiley \& Sons, New York, NY, USA, 3rd edition, 2003. 


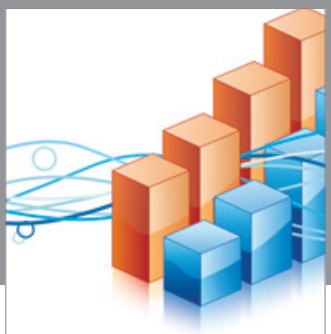

Advances in

Operations Research

vatem alat4

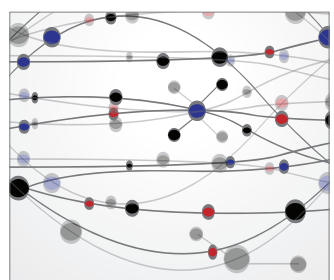

\section{The Scientific} World Journal
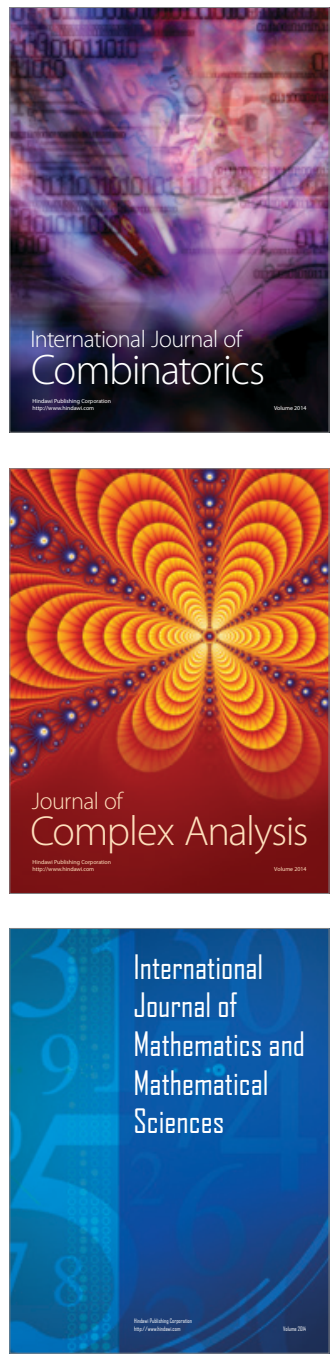
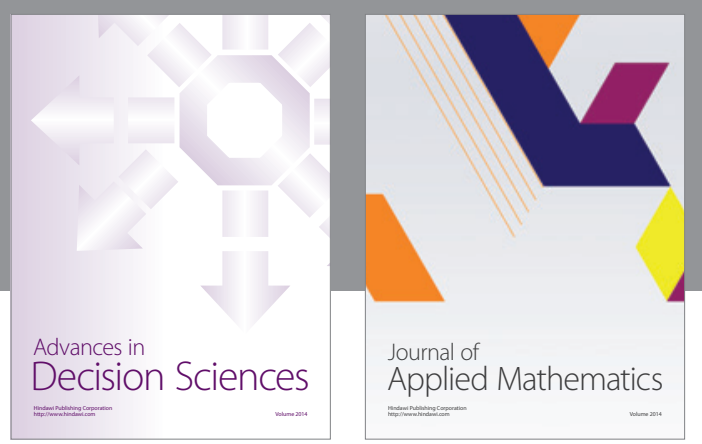

Algebra

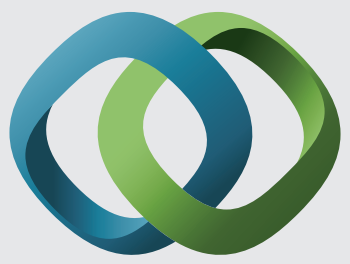

\section{Hindawi}

Submit your manuscripts at

http://www.hindawi.com
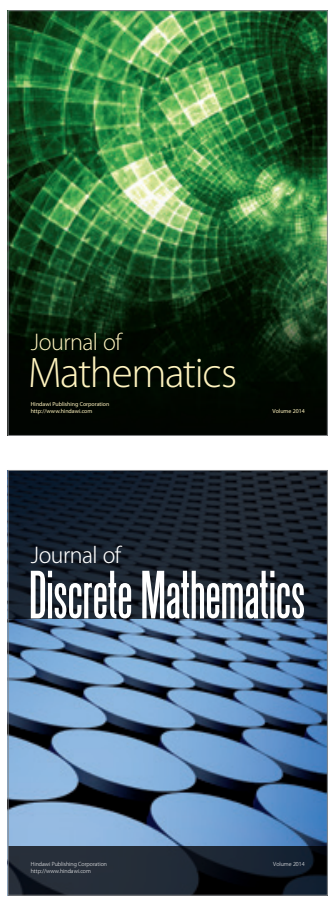

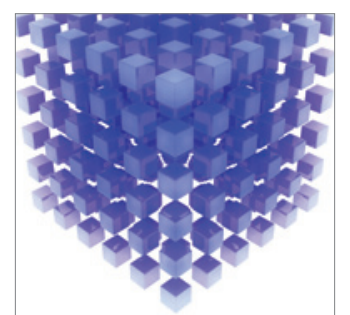

Mathematical Problems in Engineering
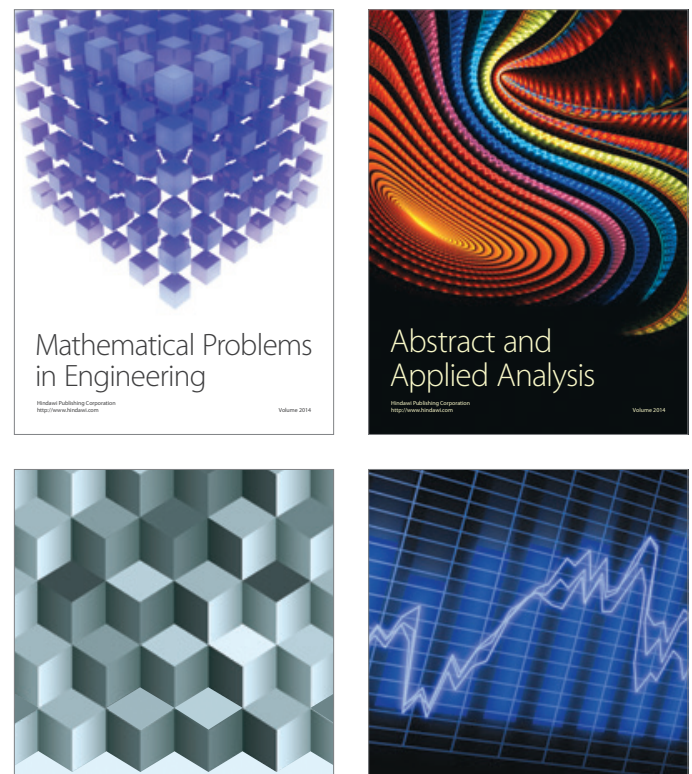

Journal of

Function Spaces

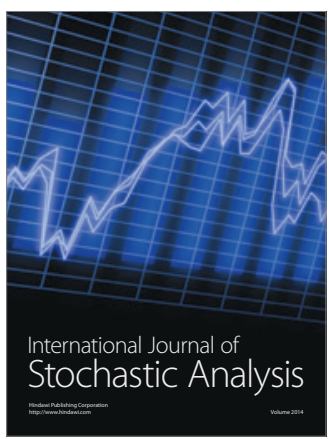

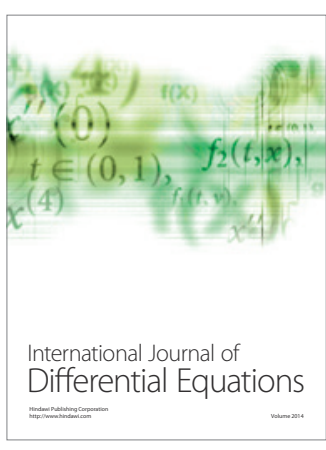
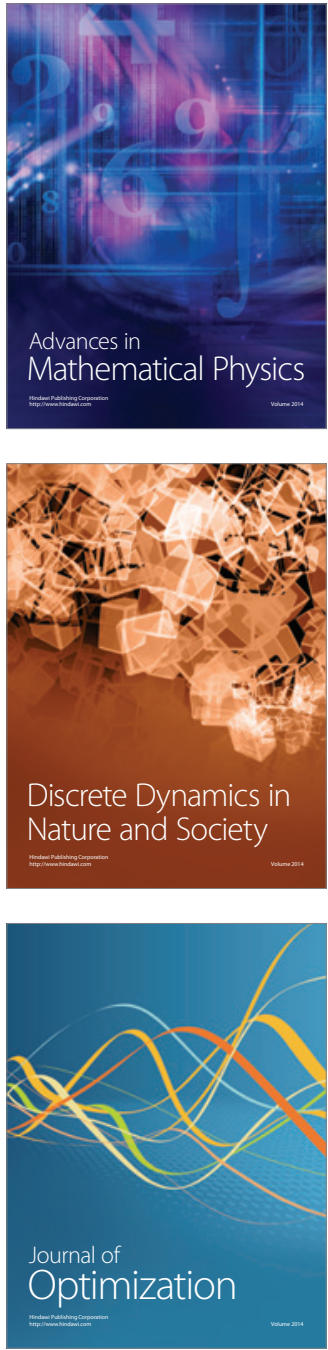